\title{
Responses of Tonically Active Neurons in the Primate's Striatum Undergo Systematic Changes during Behavioral Sensorimotor Conditioning
}

\author{
Toshihiko Aosaki, ${ }^{1,2}$ Hiroshi Tsubokawa, ${ }^{1}$ Akihiro Ishida, ${ }^{1}$ Katsushige Watanabe, ${ }^{1}$ Ann M. Graybiel, ${ }^{2}$ and \\ Minoru Kimura ${ }^{3}$ \\ 'Jichi Medical School, Department of Physiology, Minamikawachi-machi, Kawachi-gun, Tochigi 329-04, Japan, \\ ${ }^{2}$ Massachusetts Institute of Jechnology, Department of Brain and Cognitive Sciences, Cambridge, Massachusetts 02139, \\ and ${ }^{3}$ Osaka University, Faculty of Health and Sport Sciences, Machikaneyama, Toyonaka, Osaka 560, Japan
}

The basal ganglia have been implicated in motor planning and motor learning. In the study reported here, we directly tested for response plasticity in striatal neurons of macaque monkeys undergoing Pavlovian conditioning. To focus the study, we recorded from the tonically active neurons (TANs) of the striatum, which are known to respond to conditioned sensory stimuli that signal reward delivery and elicit behavioral reactions. The activities of 858 TANs were recorded extracellularly from the striatum in alert behaving macaque monkeys before, during, and after the acquisition of a classical conditioning task. Two monkeys were trained to lick reward juice delivered on a spoon simultaneously with the presentation of a click. Almost no licks were triggered by the cues at the start of training, but by the fifth day more than $90 \%$ of licks were triggered, and values were near $100 \%$ for the remainder of the 3 week training period. In the striatum, only a small number of TANs responded to the clicks at the start before conditioning (about 17\%). During training, the numbers of responding TANs gradually increased, so that by the end of training more than $50-70 \%$ of the TANs recorded (51.3-73.5\%) became responsive to the clicks. The responses consisted of a pause in firing that occurred $\sim 90$ msec after the click and that was in some cells preceded by a brief activation and in most cells was followed by a rebound excitation. Prolonged recordings from single TANs $(n=6)$ showed that individual TANs can acquire a conditioned response within at least as short a time as 10 min. TANs retained such responsiveness after overtraining, and also after a 4 week intermission in training. When the monkey was trained to receive rewards in relation to a new conditioning stimulus, TANs were capable of switching their sensory response to the new stimulus. Histological reconstruction showed that the TANs that became responsive were broadly distributed in the region of striatum explored, which included the dorsal half to two-thirds of the caudate nucleus and putamen over a large anteroposterior span. We conclude that, during the acquisition of a sensorimotor as-

\footnotetext{
Received July 2, 1993; revised Oct. 21, 1993; accepted Nov. 24, 1993.

We thank Mr. H. F. Hall, who is responsible for the photography, and Mr. G. Holm and Miss D. Major, who carried out the histology. This work was supported by the Human Frontier Science Program and Javits Award NIH R01 NS25529.

Correspondence should be addressed to Professor Ann M. Graybiel, E25-618 M.I.T., Cambridge, MA 02139.

Copyright (C) 1994 Society for Neuroscience $0270-6474 / 94 / 143969-16 \$ 05.00 / 0$
}

sociation, TANs widely distributed through the striatum become responsive to sensory stimuli that induce conditioned behavior. This distributed change in activity could serve to modulate the activity of surrounding projection neurons in the striatum engaged in mediating learned behavior.

IKey words: tonically active neurons, putamen, caudate nucleus, striatum, monkey, single-unit recording, basal ganglia, learning, conditioning, sensorimotor]

It has long been suggested that the basal ganglia are involved in the planning and execution of voluntary movements. Evidence from clinical and behavioral studies supports this view, and has emphasized that the basal ganglia may participate in the acquisition and retention of motor skills (Cools, 1980; DeLong and Georgopoulos, 1981; Evarts et al., 1984; Carli et al., 1985; Seitz et al., 1990). Neuronal response plasticity has been demonstrated in the dopamine-containing neurons of the midbrain of primates engaged in behavioral learning (Ljungberg et al., 1992; Schultz et al., 1993). There have, however, been no direct tests of behaviorally linked neuronal plasticity in the basal ganglia themselves. We therefore trained macaque monkeys on a sensorimotor conditioning task and recorded the behavior of striatal neurons as the monkeys acquired behavioral conditioning.

We chose to concentrate our recordings on the tonically active neurons (TANs) of the striatum. These TANs do not fire in rclation to body movements per se, but do specifically respond to conditioned sensory stimuli in highly trained monkeys ("type I" neurons of Kimura, 1986, 1992; Kimura et al., 1984; Apicella et al., 1991). The responses of the TANs appear to be specifically related to the conditioned significance of the sensory stimuli, because they disappear during extinction trials in which reward is withheld. The cellular phenotype of the TANs is unknown, but they are presumed to be striatal interneurons, because they are not activated by antidromic stimulation from the globus pallidus (Kimura et al., 1990). Their tonic firing $(2-10 \mathrm{~Hz})$, lack of activation in relation to movement, and apparent sparse distribution contrast sharply with the low spontaneous discharge rates $(0.01-1 \mathrm{~Hz})$, movement-related bursts of firing, and dense packing of striatal projection neurons ("type II" neurons of Kimura, 1986).

Our goals were to analyze the activity of TANs during the acquisition phase of a sensorimotor conditioning task and to test for the maintenance of acquired firing patterns during pro- 
longed training after a several-week-long break in training and after training on a new, but related, task. Our findings, briefly reported elsewhere (Aosaki et al., 1993), suggest that TANs progressively acquire responsiveness to the conditioning stimulus as the monkey learns to respond to it behaviorally, that TANs maintain their responsiveness even with overtraining and after gaps in training, and that they are capable of acquiring a response to a new stimulus if the monkey learns a new conditioning task. These findings suggest that during sensorimotor learning, the sensory responses of a class of striatal neurons undergo a change reflecting the reward value of the stimuli. This plasticity of responsiveness may help to organize neural activity of the striatum during behavioral acts.

\section{Materials and Methods}

Two male juvenile monkeys (Macaca fuscata) weighing $5.3 \mathrm{~kg}$ (monkey $\mathrm{K}$ ) and $6.3 \mathrm{~kg}$ (monkey D) were trained to perform in a behavioral task. They were fitted with intramuscular electrodes and two recording chambers. Daily single-unit recordings were made in the caudate nucleus and putamen of both hemispheres before and during acquisition of a sensorimotor association task in which click (or light) stimuli were paired with liquid reward.

In monkey $\mathrm{R}$, recordings were made in the striatum of the right hemisphere before ( $13 \mathrm{~d})$ and then during $(27 \mathrm{~d})$ conditioning with click stimuli (Fig. 1A). Then, the recording was switched to the left striatum. During the ensuing recording sessions, the monkey was trained, in parallel, with the click stimuli and also with LED light stimuli predictive of liquid reward ( $44 \mathrm{~d}$ ). The LED trials were given at irregular intervals during every day of recording. On some days there were as many LED as click trials, but on most days more click trials were delivered. After a 4 week intermission in training the right striatum was again examined with both kinds of stimuli (14 d).

In monkey D, TANs in the striatum of each hemisphere were examined before conditioning with click stimuli (right, $12 \mathrm{~d}$; left, $13 \mathrm{~d}$ ). After a 4 week behavioral training period, the activity of TANs in the striatum in each hemisphere (right, $60 \mathrm{~d}$; left, $30 \mathrm{~d}$ ) was examined with both the click and novel LED light stimuli. After a 4 week intermission, recordings were again made with both stimuli in the right striatum (26 d). A new sound (wcak click) stimulus was presented with reward to monkey $\mathrm{R}$ while original click sound was presented without reward (NEW CONDITIONING task, Fig. 1) to determine the change in the activity of TANs (right striatum, $13 \mathrm{~d}$ ). After completion of the experiments, electrode penetrations were reconstructed in serial sections through the striatum. All experimental procedures were carried out according to guidelines adopted by the American Physiological Society.

Behavioral paradigm. The monkeys were trained to sit in a primate chair situated in a soundproof room for $3-4 \mathrm{hr}$ each weekday (for up to $7 \mathrm{~d}$ /week for monkey R's initial learning). The chair allowed free movements of both arms, limited excursions of the legs, and some postural adjustments. Each monkey was trained to associate the presentation of auditory clicks (or red LED light later in the experiments) with a liquid reward (droplets of water or diluted apple juice) delivered on a spoon in front of its mouth. Their heads werc firmly fixed so that they could not see the juice drop onto the spoon. During recording sessions, reward was delivered through a peristaltic pump controlled by a computer (NEC PC9801 VX). Licking movements were monitored by a strain gage attached to the spoon and by intramuscular electrodes implanted in the muscles of the throat (earlier experiments) or tongue (later experiments). Following each recording session, the monkeys were returned to their home cages and were given juice. They were deprived of fluid on weekdays only, and were given free access to food every day. Their weights were monitored and maintained above $90 \%$ of preexperimental levels.

Before training on the conditional sensorimotor association, sensory signals were emitted at irregular 3-7 sec intervals without reward delivery so that neuronal responses to sensory signals per se could be investigated (Fig. $1 B$ ). During these sessions $(12-13 \mathrm{~d}$ ), the monkeys sat listening, and rewards were given manually during intermissions in the click delivery by use of an injection syringe connected to the spoon by a lube. A recording session consisted of $20-45$ presentations of a given type of auditory signal. When possible, various kinds of auditory stimuli, such as loud clicks, fainter clicks with slightly lower pitch, and buzzer sounds were presented while recording the activity of each neuron. Occasionally, recordings of bird songs, murmuring of a stream, sounds of waves, chirps of insects, or music were presented to mask the click stimuli.

During conditioning sessions (27-60 d), the auditory signals were emitted under computer control simultaneously with pulses of less than $300 \mathrm{msec}$ duration that activated the peristaltic pump delivering liquid reward. The pump was outside the soundproof room and was insulated to prevent the monkeys from hearing noises made by it. In monkey $R$, recordings were made nearly every day during the conditioning period (27 d) in order to investigate the time course of the changes in ncuronal responsiveness to sensory stimuli and the licking behavior until the responses reached plateau levels. Loud click sounds produced by a solenoid valve were delivered for conditioning stimuli. In monkey D, neuronal responses to the click stimuli were recorded before the conditioning period, but no recordings were made until he responded quickly and reliably to the stimuli, about 4 weeks after the start of conditioning. After acquisition of the conditional behavior, neuronal activity and licking movements were examined for more than 2 months of "overtraining." Later in the conditioning period, a red LED light on a panel was introduced as a second conditioning stimulus in parallel with the clicks. To test whether the neuronal responses acquired during the conditioning period could be maintained after a several week intermission, we stopped training the monkeys for 4 weeks, gave them free access to juice and food, and then resumed the recording scssions (14$26 \mathrm{~d})$.

During the recording periods, NO REWARD tasks and NO CUE tasks were occasionally imposed on both monkeys (Fig. 1B). In the NO REWARD tasks, sensory signals were given without reward during a recording session, so that the dependence of the neuronal responses on reward could be assessed. In the NO CUE task, no sensory cues were given, but rewards were delivered during the recording session. In this way, the relation between the neuronal responses and the licking movements could be assessed.

In monkey $\mathrm{R}$, a NEW CONDITIONING task was imposed after the conditioning period was completed (Fig. $1 B$ ). Whereas familiar loud click sounds were presented with liquid reward during the conditioning period, no reward was given in association with these sounds during the NEW CONDITIONING task. Instead, different (faint, lower pitched) clicks were associated with the reward stimuli. Stimuli of the two kinds were presented randomly during recordings. In this NEW CONDITIONING task session (13 d), recordings from single TANs were made for as long a time as possible in order to study the change in activity of individual TANs in the striatum during training.

Electrophysiological procedures. After being habituated to the behavioral condition, each monkey was deeply anesthetized with sodium pentobarbital $(25 \mathrm{mg} / \mathrm{kg})$ and was prepared for intracranial surgery. With sterile precaution, two stainless steel chambers were implanted stereotaxically and fixed to the skull to permit recording from the striatum of each hemisphere. The dura mater was left intact. Two bolts were implanted into dental cement surrounding the wells for later head fixation. Teflon-coated multistranded steel wires were implanted in the tongue or throat muscles and were led subcutaneously to the head implant. Following surgery the animals were treated with Ampicillin (150 $\mathrm{mg} / \mathrm{kg}$, s.c.) and Cephalexin $(15 \mathrm{mg} / \mathrm{kg}$, s.c.) and were allowed to recover for $4 \mathrm{~d}$ before recording.

The activity of single neurons was recorded extracellularly with glassinsulated elgiloy microelectrodes (Suzuki and Azuma, 1976) inserted through the chamber at an angle of $42-45^{\circ}$ from the vertical axis to permit access to either the putamen or the caudate nucleus. It was possible in most cases to identify which of these nuclei the electrode was in at the time of recording, because at this angle we could record characteristic high-frequency $(50-100 \mathrm{~Hz})$ pallidal activity at the end of the electrode tracks only when they passed through the putamen. Signals from the electrodes were amplified, filtered, and detected through an amplitude window discriminator by conventional electrophysiological techniques, and they were sampled by a computer (NEC PC9801 VX). Electromyographic (EMG) signals were recorded from the digastric or tongue muscles at a sampling rate of $100 \mathrm{~Hz}$ by means of chronically implanted Teflon-coated stainless steel wire electrodes. Al the beginning of the experiments in each monkey, EMG activity was recorded from the digastric muscles, but the chronically implanted electrodes became gradually insensitive, so that in the later experiments we switched to the tongue muscles, which provided much stabler signals. The signals 
A

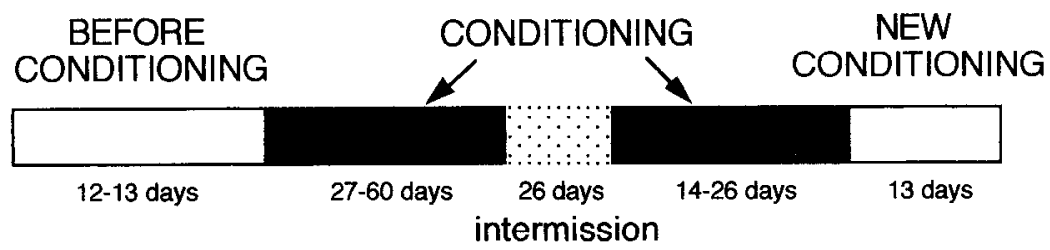

B

recording session

\section{BEFORE CONDITIONING}

click

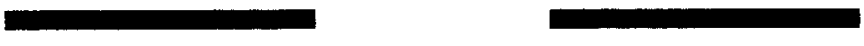

reward

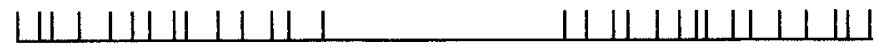

recording session

click

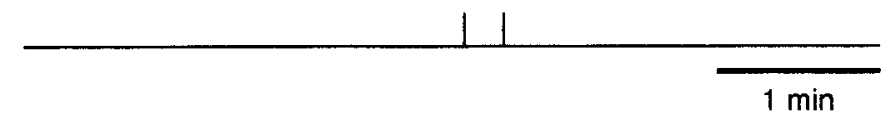

reward

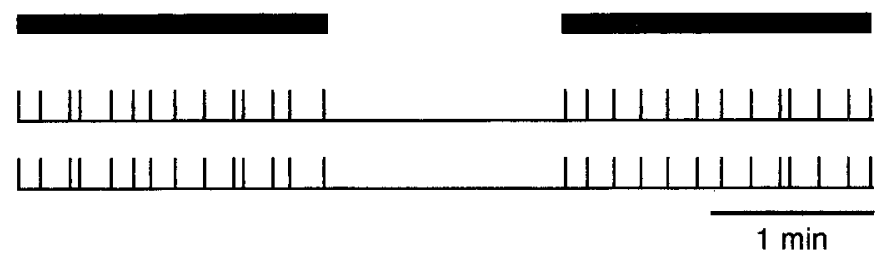

\section{CONDITIONING}

\section{recording session \\ click}

reward

\section{NO REWARD}
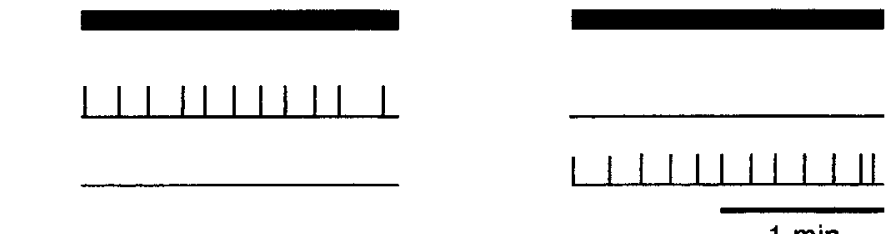

$1 \mathrm{~min}$

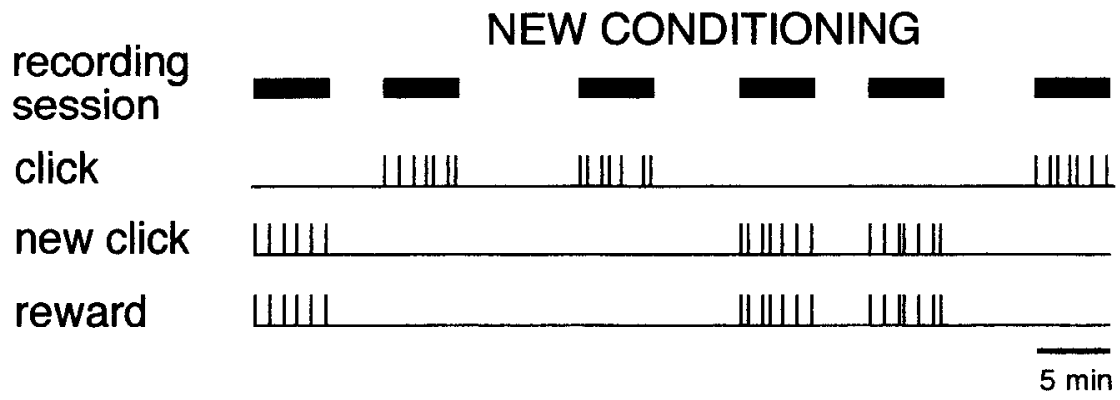

Figure 1. Experimental paradigm. $A$, Recordings were made from tonically active neurons (TANs) in the caudate nucleus and putamen in one or both monkeys before conditioning (12-13 d), during conditioning (27-60 d), after a $26 \mathrm{~d}$ break in training (14-26 d), and during a second conditioning task with a new sensory cue (13d). $B$, Before conditioning, clicks and reward were given independently. Recordings were made during presentation of clicks. One recording session consisted of 30-45 trials (clicks). During conditioning, clicks and reward delivery were simultaneously presented. Outflow of the reward juice onto the spoon lasted for about $150 \mathrm{msec}$. After overtraining, the characteristics of the activity of TANs were further examined during occasional presentations of LED lights, clicks with no reward (NO REWARD task), or by presentations of reward delivery without clicks (NO CUE task). In the NEW CONDITIONING task, the original conditioning clicks were given without reward, but novel clicks were given with reward delivery. were then amplified, rectified, integrated, and monitored on line, simultaneously with neuronal activity, on a computer display. Licking movements were also recorded by a strain gage pasted on the spoon at a sampling rate of $100 \mathrm{~Hz}$ and displayed on line on an oscilloscope. The behavior of the animals was routinely monitored by a video camera.

All electrical pulses from neuronal discharges and EMG activity were sampled and displayed on line in the form of peristimulus time histograms and integrated line traces on the computer display. The computer controlled the experimental trial schedule, and the recording was canceled if electrical noise or noise artifact due to jerky movements by the animals contaminated the data. Off-line computer programs were used for data analysis. Statistical evaluation of changes in neuronal firing rate was carried out by the two-tailed Wilcoxon test for matched pairs. Responses to the click stimuli were defined as increases or decreases in impulse discharge rate after presentation of the sensory stimulus relative to discharge rates before stimulation. The onset time of a response was defined by the first of three or more consecutive $15 \mathrm{msec}$ bins in which activity deviated from the average value during the control period. The offset time was defined by the first of three or more consecutive bins in which activity returned to control levels. Differences were considered significant at $p \leq 0.01$ (Kimura, 1986).

Histological reconstruction. At the end of recording, the monkeys were lightly anesthetized with ketamine hydrochloride $(30 \mathrm{mg} / \mathrm{kg}$, i.m.), and several small electrolytic marking lesions were placed in each hemisphere to mark the recording level by passing a positive current through the microelectrode $(5-15 \mu \mathrm{A}$ for $2 \mathrm{~min})$. The animals were then deeply anesthetized with an overdose of pentobarbital $(>70 \mathrm{mg} / \mathrm{kg}, \mathrm{i} . \mathrm{m}$.) and were perfused through the left ventricle with $4 \%$ paraformaldehyde in $0.1 \mathrm{~m}$ phosphate buffer. Blocks through the striatum were cut at $50 \mu \mathrm{m}$ in the transverse plane and were stained with cresylecht violet. Re- 


\section{EMG}

\section{BEFORE CONDITIONING}

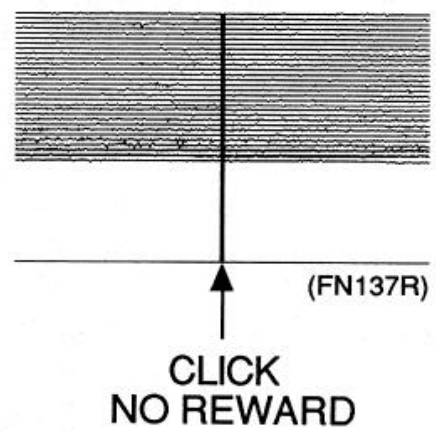

\section{CONDITIONING}

DAY 2

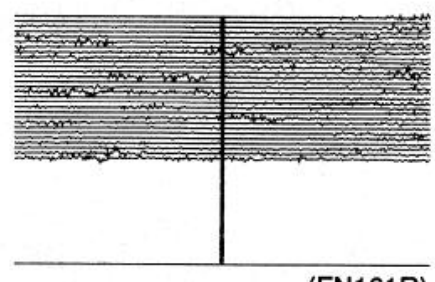

DAY 5

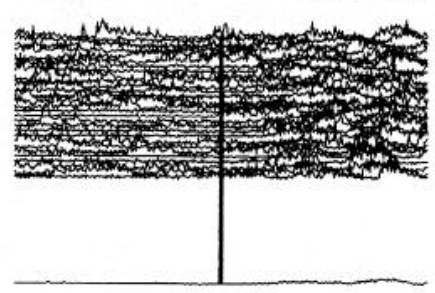

(FN206R)

DAY 10

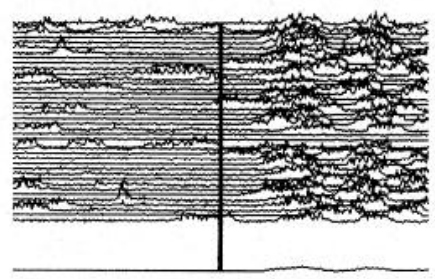

(FN277R)

DAY 21

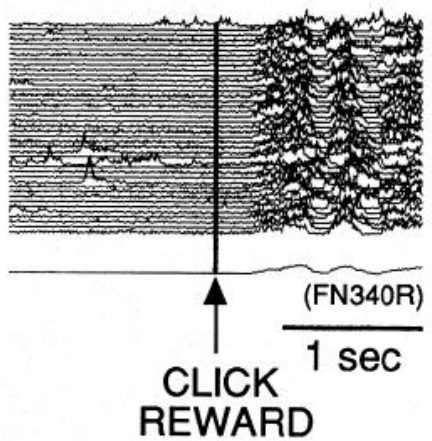

Figure 2. Change in licking behavior in monkey $\mathrm{R}$ before and during learning. Records of the electromyographic (EMG) activity of the digastric muscles were sequentially aligned at the time of click presentations. An averaged EMG trace is shown below each set of traces. In this and all succeeding figures, the first trace is at the top, and the last trace at the bottom. The file name of the cell is shown in parentheses.
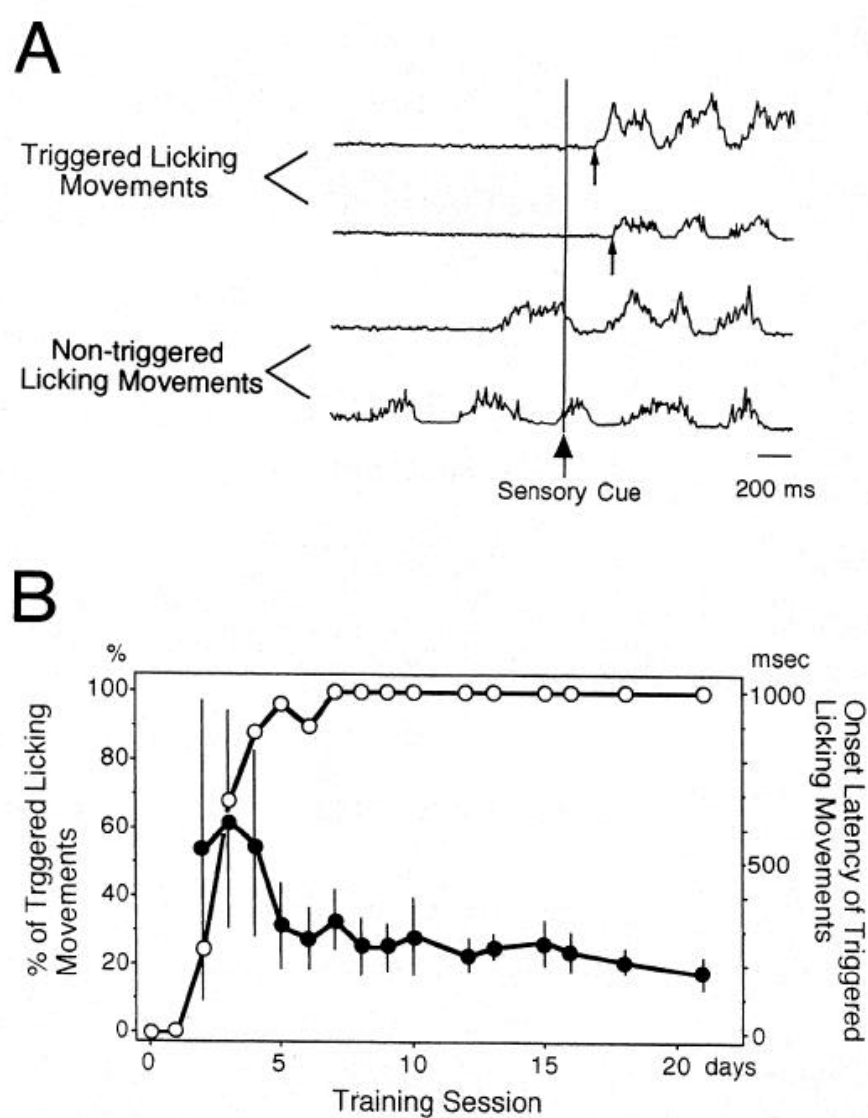

Figure 3. Classification of licking movements and increase in clicktriggered licking movements during learning. $A$, Licking movements were defined as triggered when they started after the click stimulus (see small arrows) and as nontriggered when they started before the click stimulus. The large arrow indicates the time of click presentation. $B$, Open circles show percentage of triggered licking movements plotted against time for one recording session in which the monkey showed his best daily performance. Solid circles show change in mean onset latency of the triggered licking movements in the same recording session.

cording tracks were reconstructed by reference to marks from the electrolytic lesions, and the positions at which neuronal activity was recorded were estimated by reference to the distances from the micrometer readings made at the time of recording, corrected for shrinkage resulting from the fixation procedure, and expressed in approximate HorsleyClarke coordinates (Kusama and Mabuchi, 1970).

\section{Results}

\section{Behavior}

During the preconditioning sessions, when liquid reward was given independently of sensory signals, the monkeys noticed, apparently by chance, that the juice was delivered on the spoon in front of their mouths, and they paid attention to the spoon by making exploratory movements of the throat or tongue.

In monkey $\mathrm{R}$, the behavioral change during learning was examined almost daily from the start of sensorimotor conditioning until it reached the plateau phase. Figure 2 shows examples of licking behavior at different times during the conditioning task. Before conditioning, when no juice reward was given with the click sounds, the monkey made almost no licking movements. On the second day of conditioning, the monkey licked the spoon almost all the time; his behavior was indistinguishable from the exploratory movements before conditioning. By the fifth day, he tended to lick after the clicks, and this behavior became more 

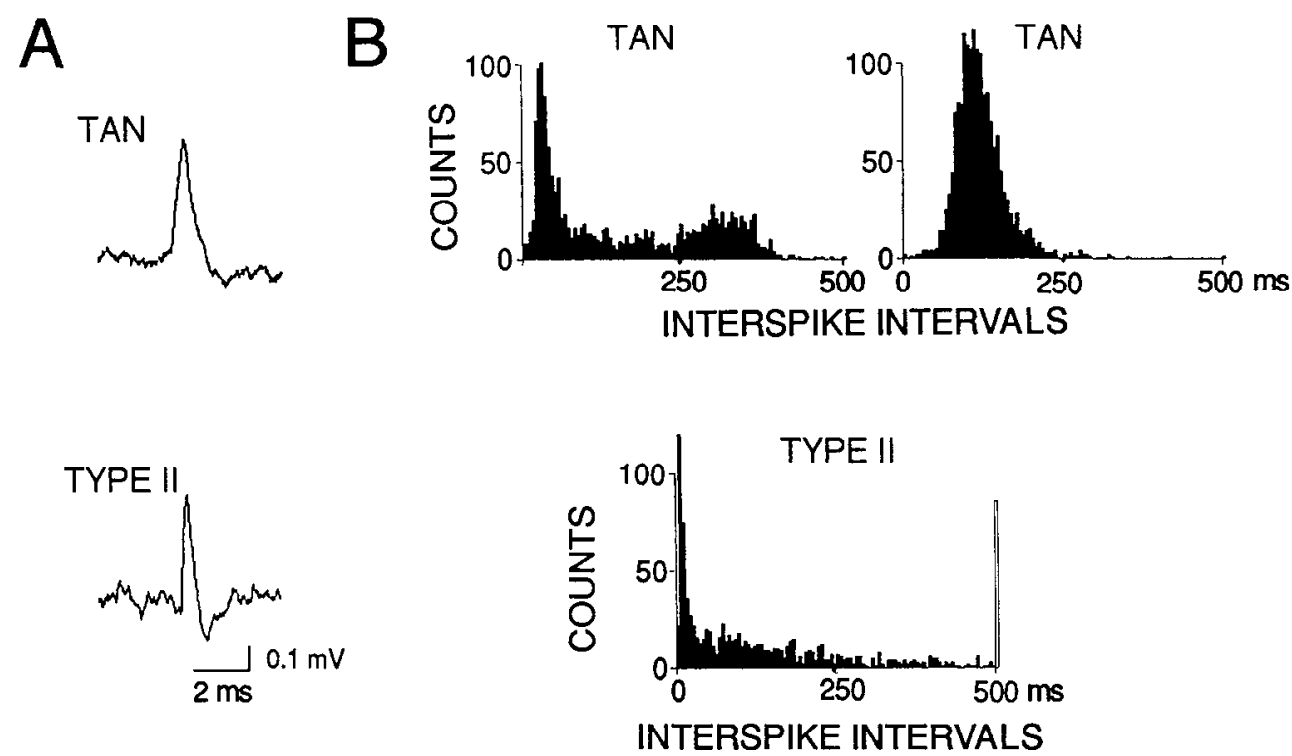

Figure 4. Criteria for identification of tonically active neurons (TANs). TANs fire tonically with a spike of long duration at a frequency of $2-10 \mathrm{~Hz}(A$ and $B, T A N)$, often with one or two peaks in their interspike-interval histograms. A second type of striatal neuron (type II), presumed to correspond to medium-sized spiny projection neurons, exhibits mostly very low frequency $(<2$ $\mathrm{Hz}$ ) firing with a shorter spike duration $(A, T Y P E I I)$ and one interspike interval peak at less than $1 \mathrm{msec}(B, T Y P E$ II). marked by day 10 . By day 21 , the onset of the licking movements was time-locked to the click sounds.

In order to quantify this behavioral change, we classified the licking behavior into two categories of licking movements, triggered and nontriggered (Fig. $3 A$ ). The onset of the licking movements in each trial was examined on each EMG trace taken from the digastric or tongue muscles (for Fig. $3 \mathrm{~A}$, digastric), and, if it occurred after the presentation of the click, the licking was considered to be movement triggered by the cue. The licking movements were considered to be nontriggered if they occurred before the sensory cue. The percentage of triggered licks in a recording session was used as an index of the conditioned behavior.

Figure $3 B$ (open circles) summarizes the time course of the behavioral change in monkey $\mathrm{R}$. Almost no triggered licks were observed on days 1 and 2, but the animal learned very quickly on days 3 and 4 , and achieved a performance of more than $80 \%$ by day 4 and a level of more than $90 \%$ correct by day 5 . The onset latency of the triggered licks from the time of sensory signals was also measured, and was plotted against time. As shown in Figure $3 B$ (solid circles), near the beginning of training, the mean latency of licking was greater than $530 \mathrm{msec}(534.3$ \pm 438.8 and $624.8 \pm 316.0 \mathrm{msec}$, mean $\pm \mathrm{SD}$, on days 2 and 3 , respectively) and then decreased gradually to less than 180 msec by day $21(175.3 \pm 47.8 \mathrm{msec})$. The SDs of the onset latencies also decreased markedly during training.

\section{Identification of tonically active neurons}

The activity of 858 TANs was recorded extracellularly from the two monkeys before and during sensorimotor conditioning. It was not difficult to discriminate the TANs from the more abundant type II neurons of the striatum. The TANs discharged negative impulses with long spike durations $(1.8-5.0 \mathrm{msec})$ at low frequencies $(2-10 \mathrm{~Hz})$, whereas the type II neurons were usually quite silent and were activated only phasically with spikes of shorter duration $(<1.2 \mathrm{msec}$; Fig. $4 A$ ). The mean frequency of firing of the type II neurons was less than $2 \mathrm{~Hz}$. In our sample, the tonic irregular discharge of the TANs averaged $4.7 \pm 1.1$ $\mathrm{Hz}(2.1-8.7 \mathrm{~Hz}$, data from $n=595)$. Data that were contaminated by noises or artifacts were omitted in this frequency anal- ysis. Interspike-interval histogram analysis (Fig. 4B) showed that the TANs had different discharge patterns, in the extreme having two peaks (roughly estimated at 30 and $320 \mathrm{msec}$ ) or one peak at about $130 \mathrm{msec}$. Occasionally, individual TANs showed these two different firing patterns alternately during recording. The interspike-interval histograms for the type II neurons had a single peak close to $0 \mathrm{msec}$.

\section{Histological reconstruction of TANs sampled}

The TANs were usually encountered at intervals of 400-500 $\mu \mathrm{m}$ along electrode penetrations both in the caudate nucleus and in the putamen. We sampled the TANs in both nuclei in the right hemisphere from A1 5 to A28 in monkey $R$ and from A14 to A22 in both hemispheres in monkey D. Approximately the dorsal half to two-thirds of the nuclei were included. Quite strikingly, as shown in Figure 5, the distribution of TANs that became responsive after training was widespread both in the caudate nucleus and in the putamen.

\section{Selectivity of the response of TANs for the conditioning stimuli}

Figure 6 shows a typical example of the activity of TANs before and after behavioral conditioning. The neuronal activity is aligned on the occurrence of the click presentations both before conditioning (without reward) and after conditioning (with reward). The EMG activity associated with licking movements and the averaged EMG traces are shown in the middle and lower traces. Before conditioning, when the clicks and rewards were not associated, the monkeys did not make licking movements and most TANs (106 of 122 cells in monkey R and 148 of 183 cells in monkey D) showed no response to the clicks. By contrast, after the acquisition of the sensorimotor association, a majority of the TANs in the first conditioning task (114 of 195 cells in monkey $R$ and 118 of 179 cells in monkey D) responded to the click sound. All the responsive cells showed suppression of firing and about a fifth of the TANs showed an initial activation after presentation of the click and a rebound activation after the suppression. The TAN illustrated in Figure 6 did not exhibit the initial transient activation, but initial activation responses at $\sim 60 \mathrm{msec}$ are illustrated in the records of Figures 9,10 , and 12. All of the 114 TANs recorded in the right striatum of mon- 


\section{A BEFORE CONDITIONING}
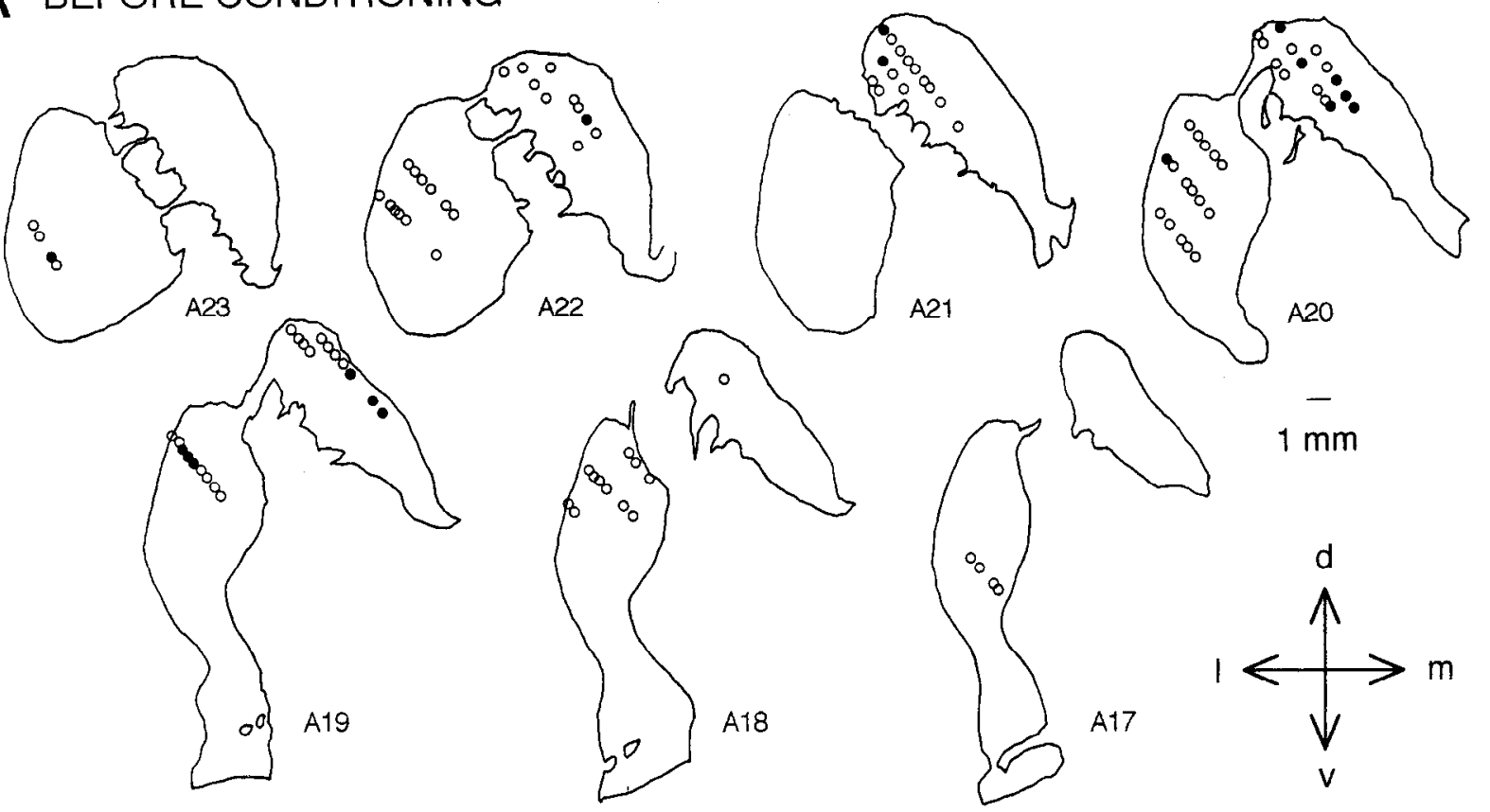

\section{B AFTER CONDITIONING}

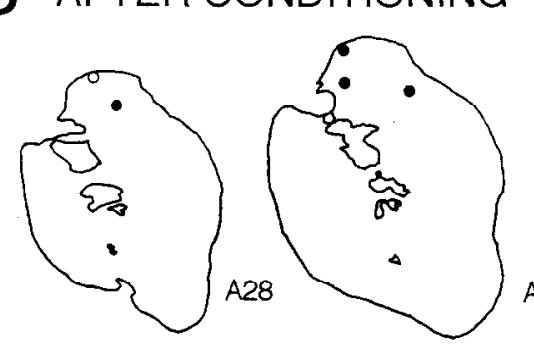

A27
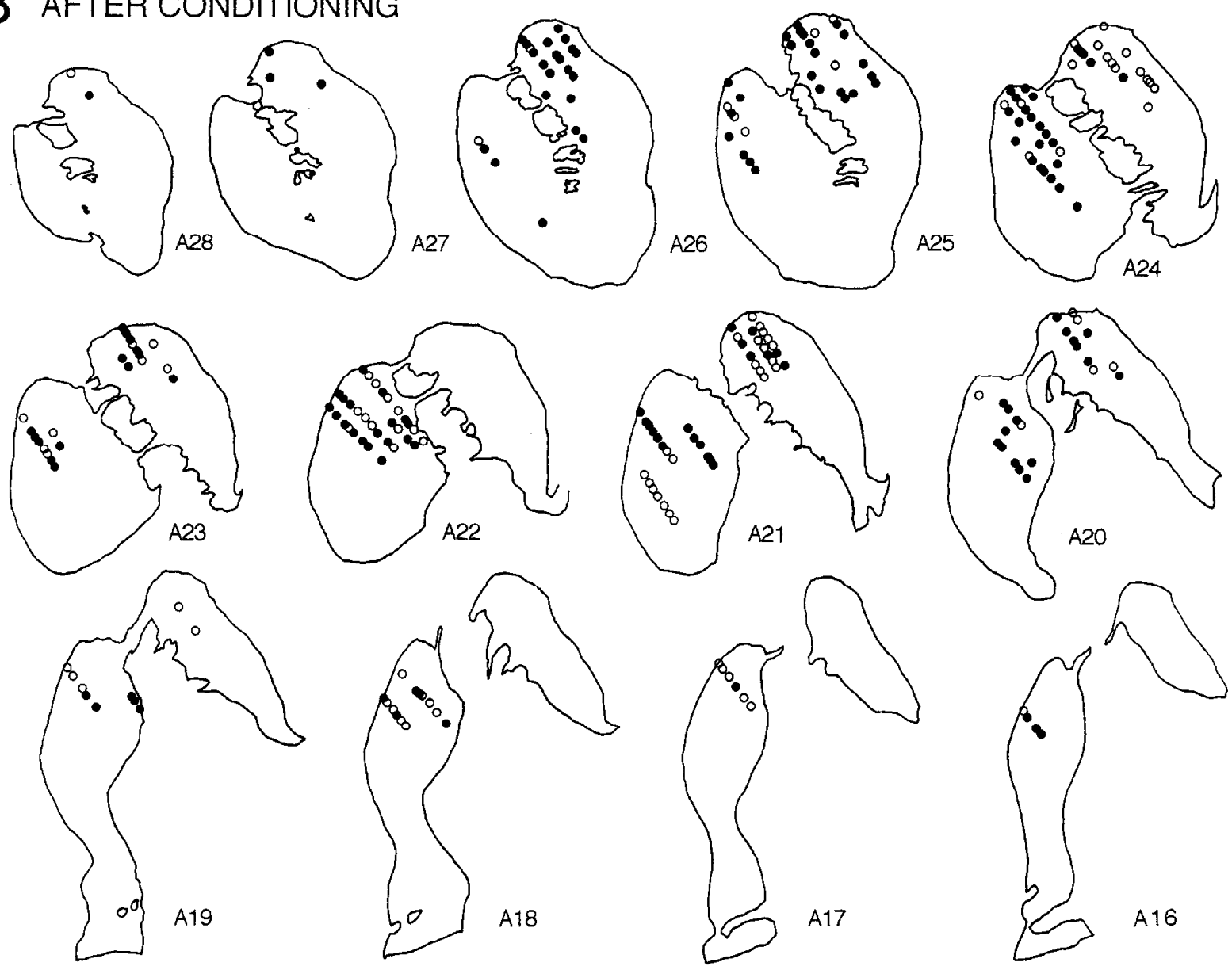

Figure 5. Histological reconstruction of TAN recording sites in the right hemisphere of the brain of monkey R. Approximate Horsley-Clarke coordinates are shown below each chart. The sites of TANs sampled before conditioning are shown in $A$ and those sampled during and after conditioning (before intermission) are shown in B. Open circles indicate the locations of TANs that showed no response to the clicks. Solid circles indicate the sites of TANs that responded to the clicks. $m$, medial; $l$, lateral; $d$, dorsal; $v$, ventral. 
key $\mathbf{R}$ that responded to the clicks after training showed suppression of firing. The TAN illustrated in Figure 6 showed a complete suppression with a latency of $90 \mathrm{msec}$ and a duration of 189 msec. Ninety-eight of the 114 responding cells in the monkey $\mathrm{R}$ right hemisphere sample showed complete suppression. In most cells the suppression was followed by a transient rebound activation. We sometimes observed an oscillatory response to the sensory stimuli, as shown in Figures 7 and 12 .

Before conditioning with reward juice, a small number of TANs (about 17\%) showed some response to the clicks. In order to test what kind of clicks were most effective to elicit the response, we presented various kinds of sounds without any reward while recording from single TANs. We found that the louder the clicks were, the more pronounced the response (Table 1). Figure 7 shows a typical example of a TAN that showed a strong response to the louder, more alerting click and a smaller response to the faint click. When we deliberately added distracting noises, such as music, sounds of waves, songs of birds, the responses to the clicks weakened (data not shown). These response patterns are consistent with the idea that the $11-21 \%$ of TANs responding before conditioning did so preferentially to stimuli that attracted the monkey's attention.

Because the licking movements made by the monkeys became closely related to the onset of the clicks, we carried out controls to test whether the response of the TANs was strictly induced by the conditioning click stimuli or whether, instead, the TANs fired in relation to the licking movements themselves. We did this in several ways. First, we compared the temporal relation of the TAN response to the clicks and to the licking. Figure 8 shows two examples of TAN firing in relation to the clicks with licking movements detected by a strain gage (Fig. 8A) and with EMG traces from the digastric muscles (Fig. $8 B$ ). Clear responses to the conditioning clicks were evident when the neuronal activities were aligned at the time of presentation of the stimuli (left panels of Fig. $8 A, B$ ). However, when neuronal activity was aligned with the time of the onset of the licking movements,
Table 1. The number of TANs that responded to loul clicks and to faint clicks before the beginning of behavioral conditioning in monkeys $R$ and $D$

\begin{tabular}{lll} 
& $\begin{array}{l}\text { Loud click } \\
\text { before } \\
\text { conditioning }\end{array}$ & $\begin{array}{l}\text { Faint click } \\
\text { before } \\
\text { conditioning }\end{array}$ \\
\hline Putamen & $31 / 201$ & $3 / 23$ \\
Caudate n. & $(15.4 \%)$ & $(13.0 \%)$ \\
Striatum & $20 / 104$ & $1 / 13$ \\
& $(19.2 \%)$ & $(7.7 \%)$ \\
& $51 / 305$ & $4 / 36$ \\
& $(16.7 \%)$ & $(11.1 \%)$ \\
\hline
\end{tabular}

significant modulation of neuronal activities was not observed either before or after licking movements (Fig. $8 A, B$, right panels). Such controls suggest that the TANs responded in relation to the sensory cues that triggered the licking movements, and not in relation to the licking movements per se.

We further tested for responses of TANs in relation to licking in the NO CUE task. Figure 9 shows one example of the eight TANs tested. This cell rapidly weakened its response when no sensory cue for reward delivery was given. But when the animals noticed that the juice came at the same intervals, they began to lick the spoon. They made many futile licking movements that failed to get them reward in the NO CUE trials, because their licking movements were not restricted to the reward delivery time. Nevertheless, licking movements in most trials were appropriatc, as shown by the averaged EMG activity of the tongue muscles. Even in these successful trials, however, the response rapidly weakened. All TANs tested showed a similar loss of response in NO CUE trials. This evidence suggests further that the response of the TANs was induced by the conditioned sensory cues and not in relation to the licking movements per se.

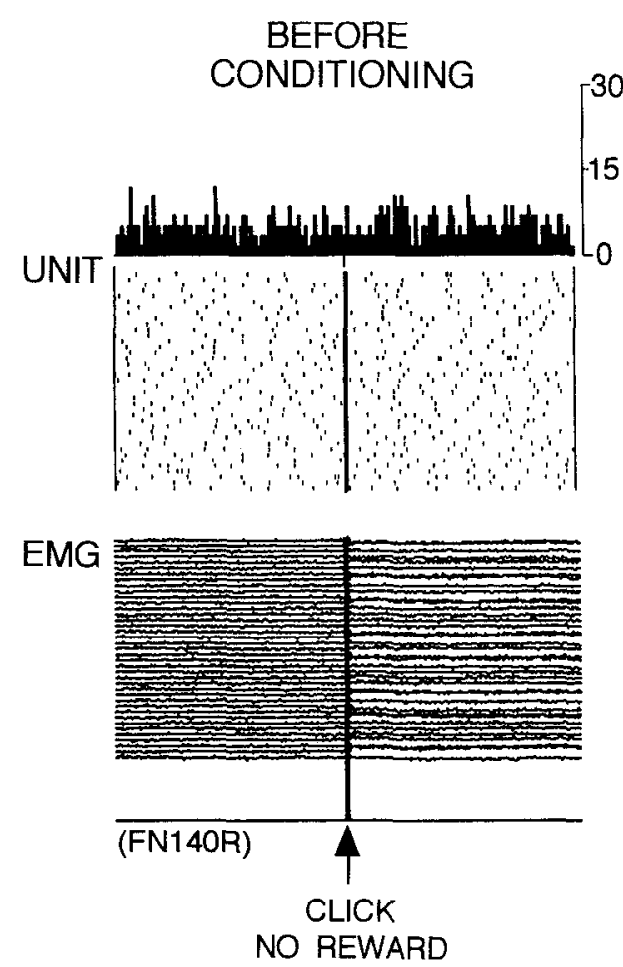

NO REWARD

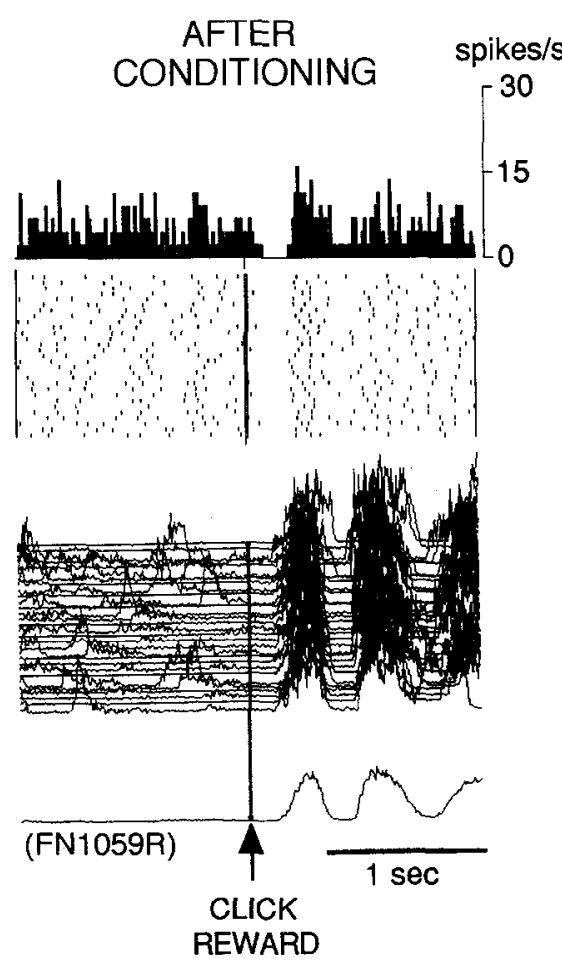

Figure 6. Activity of TANs recorded during the behavioral task before and after conditioning. Unit activity was aligned at the time of click presentation and is shown in raster-dot displays and histograms. Note that the raster plots are ordered sequentially from bottom to top in this and all succeeding figures. Electromyographic (EMG) activity of the tongue muscle, rectified and integrated, is shown in successive traces sequentially aligned from top to bottom (see Fig. 2). The averaged EMG trace is shown below individual traces. Before conditioning, no change in activity of TANs occurred in relation to the clicks, and no licking movements were made. After conditioning, many TANs responded to the clicks delivered with reward by a depression of tonic activity. In some TANs, the depression of activity was preceded by an initial excitation, not shown in this figure; such activations are shown in Figures 9 and 11. The pause in firing was followed by a rebound activation. 
LOUD CLICK

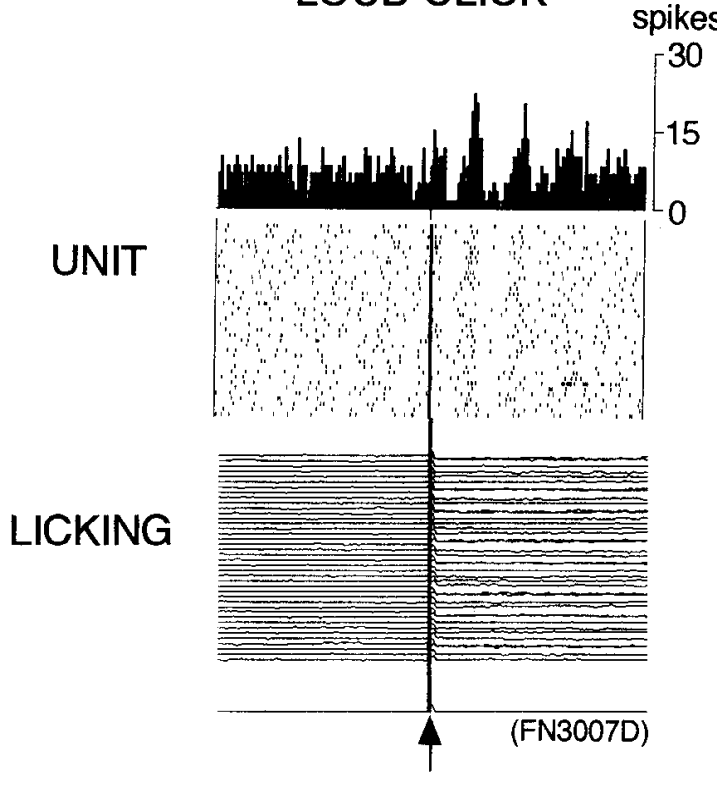

FAINT CLICK

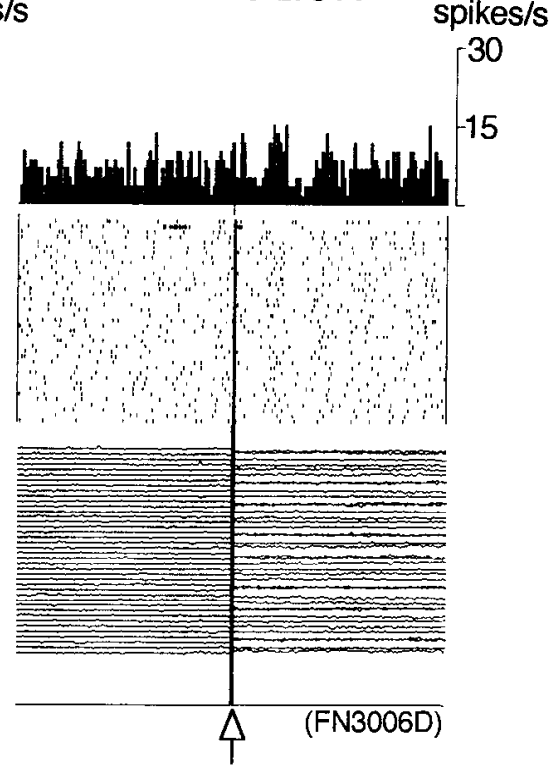

Figure 7. An example of the response pattern of the $15-20 \%$ of TANs that responded to the click stimuli before conditioning. No reward was given in association with the click sounds. This TAN showed a strong response when louder clicks were presented and a weaker response when the clicks were faint. The neuron tended to show an oscillatory response. Solid arrow indicates the time of loud click presentation, and open arrow indicates time of faint click presentation.
Other instances of dissociation of the TAN responses and licking onset were noted throughout the experiments, for example, in the extinction trials (see Figs. 12, 13B).

\section{Progressive change in responsiveness of TANs during behavioral learning}

The large majority of TANs recorded did not respond to the clicks (unconditioned stimuli) before training. To address the question of whether and how such initially nonresponsive populations of TANs could acquire responsiveness to the sensory cue during sensorimotor conditioning, we recorded the activity of as many TANs as possible in both the putamen and the caudate nucleus before and after conditioning in both monkeys, and also nearly every day during conditioning in monkey $\mathrm{R}$. The results are shown in Figures 10 and 11.

Figure $10 \mathrm{~A}$ shows peristimulus-time histograms of the population responses of TANs recorded in the striatum of monkey $\mathrm{R}$ and demonstrates a gradual increase of the responsiveness of the TANs to the click sound. Before conditioning, the histograms for the 80 cells recorded in the putamen and the 42 cells recorded in the caudate nucleus (top) showed no population response to the click, and the averaged EMGs indicated that no licking movements occurred. One to $4 \mathrm{~d}$ after the start of conditioning, the averaged activity of the 29 TANs recorded in the putamen and the 22 TANs in the caudate nucleus indicated a small decline in firing followed by a rebound activation in response to the click. After 5-8 d of training, the responses of the TANs in both nuclei ( 32 cells in the putamen and 21 cells in the caudate nucleus) were more pronounced, and a clear initial activation became visible for the first time. Licking movements had also become increasingly time-locked to the clicks. After 9-14 days, the population responses were further increased in both nuclei, and within the third week of learning, both the initial activation following the clicks and the following suppression sharpened.

The population response of the TANs in the caudate nucleus appeared somewhat stronger than that in the putamen. This bias is shown in Figure $10 B$, which illustrates the rate of increase in the ratio of responsive neurons to all TANs examined in the two nuclei. A small number of $\mathrm{T} \Lambda \mathrm{N}$ s responded to the click from the start of recording before conditioning (on day $0,11 \%$ in putamen and $16 \%$ in caudate nucleus). The percentage of responsive neurons rose rapidly in both nuclei, but was somewhat higher in the caudate nucleus than that in the putamen by the second week of learning.

\section{Retention of the acquired sensory responsiveness}

In order to determine whether the acquired responses of the TANs could be maintained after a long break of training, we stopped training the animals for about 4 weeks and let them gain free access to juice and food in their cages. After the intermission, we resumed recording. Figure 11 (stippled bars) shows that the acquired responsiveness was retained without decrement in both monkeys after the 4 week intermission. For monkey $\mathrm{R}$, the values were $58.5 \%$ (114 of 195 cells) before and $63.7 \%$ (72 of 113 cells) after the break; for monkey D, $65.9 \%$ ( 118 of 179 cells) responded before and 74.2\% (49 of 66 cells) after the break.

\section{Context dependence of the acquired sensory responsiveness}

The responses of the TANs, though stable over lapses in training, were by no means fixed even after prolonged training. As originally demonstrated by Kimura et al. (1984), the neurons that showed a strong response to the conditioning stimulus weakened their responsiveness when the sensory stimulus no longer accompanied reward delivery. Of 16 cells examined in detail, 10 showed such context dependency. However, we found that this change occurred only after many presentations of the unrewarded clicks (see Figs. 12, 13).

To determine whether individual TANs could respond to more than one kind of conditioned stimulus, we trained both monkeys $\mathrm{R}$ and $\mathrm{D}$, after their overtraining with clicks, with either clicks or LED lights as a second conditioning stimuli. We found (Table 2; T. Aosaki et al., unpublished observations) that some TANs showed responses both to clicks and to LED lights, that other TANs responded only to the clicks, and that still other TANs exhibited responses only to the lights. TANs of all three types appeared to be intermixed rather than being spatially 
A
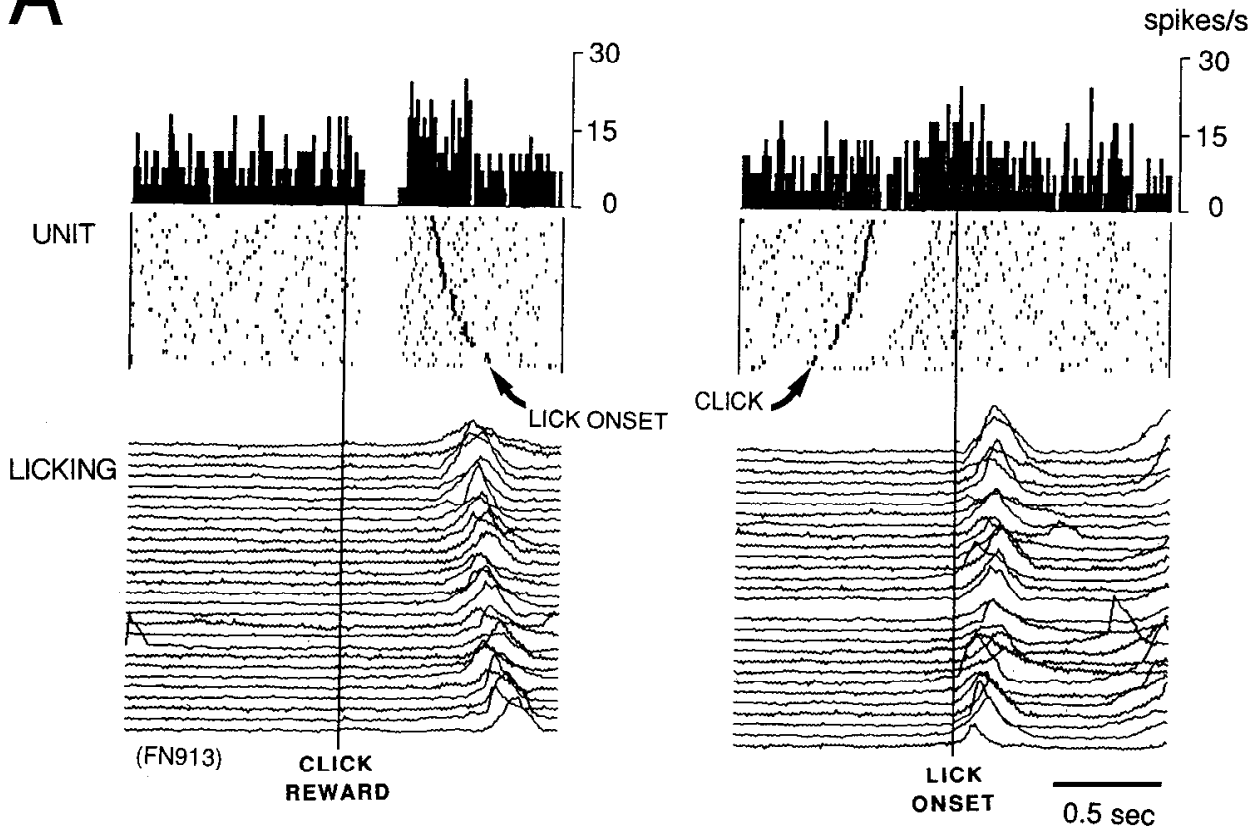

B
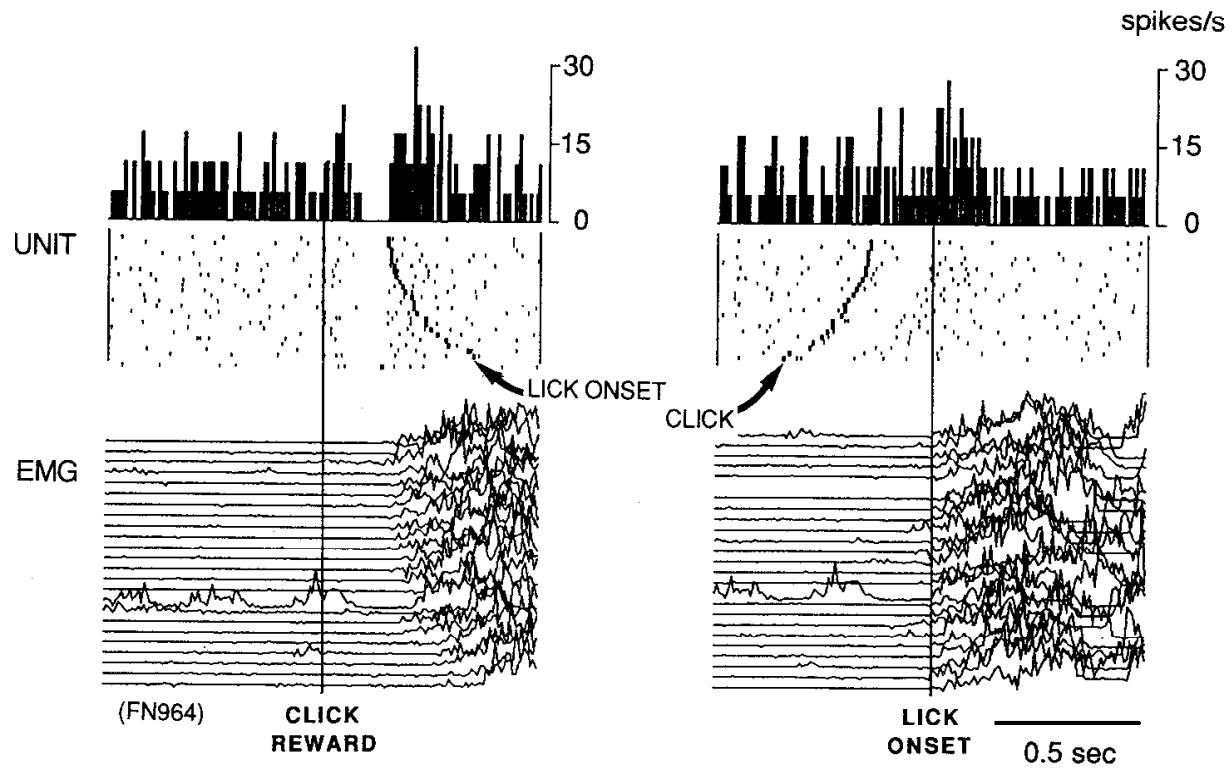

Figure 8. Lack of time-locked relation of TAN activity and licking movements. Two cxamples of TANs for which clicks elicited a strong response are shown in $A$ and $B$. In $A$, the licking movements detected by a strain gage are shown below the unit activity rasters. In $B$, EMG traces from the digastric muscles are illustrated. In both $A$ and $B$, two raster dot displays of the same activity are shown. The displays on the left are aligned at the time of presentation of clicks. The displays on the right are aligned at the onset of licking movements. The responses of the TANs are time-locked to the onset of the clicks, not to the onset of the licking movements or EMG activity.

separated (Aosaki et al., unpublished observations). Altogether, $26.8 \%$ of the neurons tested in the two monkeys ( 26 of 97 cells) responded only to clicks, $34.0 \%$ (33 of 97 cells) responded only to light stimuli, and the remaining $39.2 \%$ responded to both stimuli. These observations suggest that, in a given conditioning paradigm, some TANs can become conditioned to respond to one set of stimuli and other TANs to another set of stimuli in the task, whereas other TANs can respond to at least two different stimuli of different modalities in the same behavioral setting.

\section{Acquisition and loss of conditioned responses by individual TANS}

As a further test of the capacity of TANs to show response plasticity, we asked whether the conditioned response of TANs that had acquired a response to conditioning clicks could be switched to a new conditioning stimulus when the original stimulus was no longer accompanied by reward delivery but a new stimulus was rewarded. In the NEW CONDITIONING task, a different click sound was presented as a new conditioning stimulus associated with delivery of reward juice, and no reward was given when the familiar, original clicks were presented. Recording sessions in which the new stimuli were coupled with reward and sessions in which the original clicks were presented without reward, both containing about 30-40 stimuli, were presented alternately to monkey $\mathrm{R}$.

A total of 56 TANs were recorded during NEW CONDITIONING experiments $(13 \mathrm{~d})$. Recordings were made every day. It was only on day 4 that the TAN first showed a response to the new stimulus. For each cell, we tried to examine responses 
WITH CLICKS

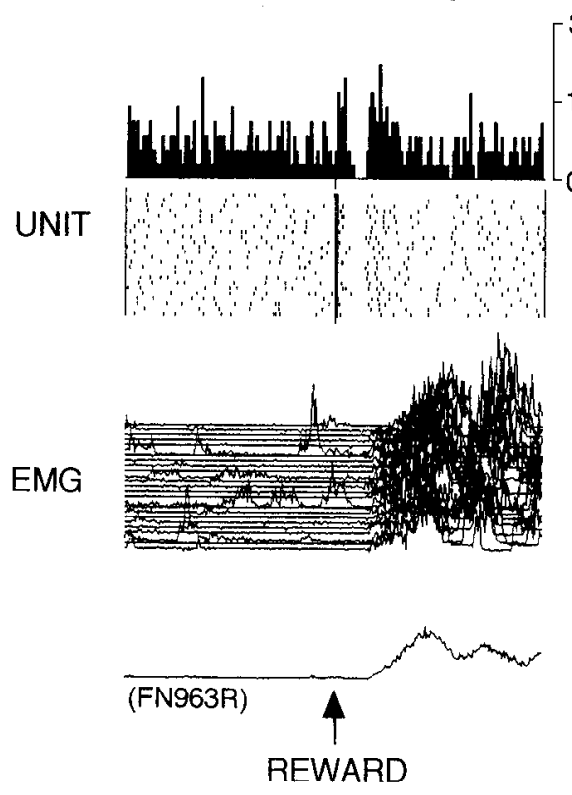

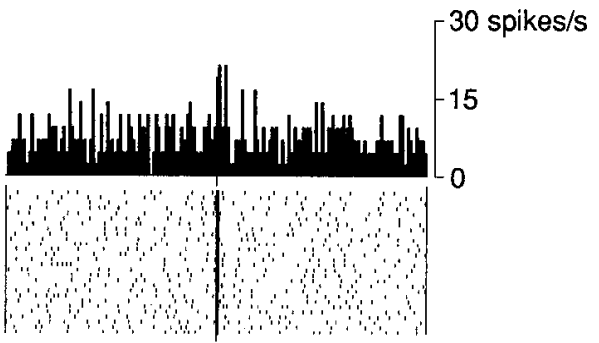

Figure 9. Evidence that the response of TANs to the sensory conditioning signal is not related to the reward itself, or to the licking movements accompanying reward consumption. Data were taken from a single TAN in the caudate nucleus. The TAN responded to the clicks after conditioning (left), but this response declined almost immediately when the clicks were not presented in the next recording period. The EMGs of the tongue muscles show that the monkey licked the spoon vigorously in most trials despite the absence of clicks.
WITHOUT CLICKS
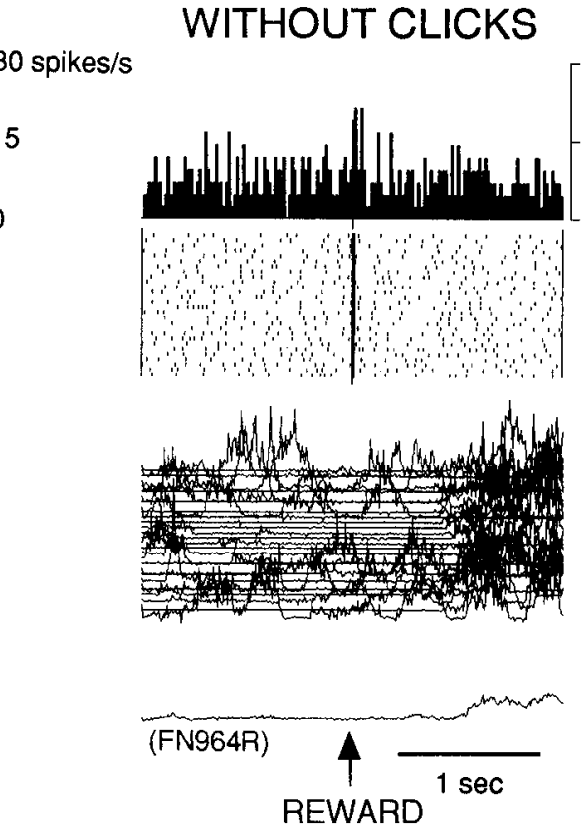

to the two kinds of clicks for the very first set of trials for that cell. We found that the responses to the original conditioning stimulus were strongly engrained. Regardless of the day of recording, a majority of cells, when first tested with both old and new clicks, showed time-locked responses only to the original clicks in spite of the lack of its association with reward. The responses of the cells changed gradually.

Figure 12 shows the activity of a TAN and the associated licking behavior on the first day of the NEW CONDITIONING task. The records illustrated were made after 240 presentations of the new click with reward and the old click without reward. At first, the monkey licked the spoon several times when the old click was presented, but he became irritated and stopped licking when he noticed that no reward came. When the new click sound was presented with reward, he at first neglected the sound and did not lick at all. But soon he began to protrude his tongue tentatively to touch the spoon during the new conditioning period and found the reward juice on the spoon. At this time in the retraining, the TAN illustrated evidenced no response to the new stimulus with reward and maintained a strong

Table 2. The numbers of TANs that responded either only to clicks, only to LED lights, or to both stimuli

\begin{tabular}{|c|c|c|c|c|}
\hline & $\begin{array}{l}\text { TANS that } \\
\text { responded } \\
\text { only to } \\
\text { click }\end{array}$ & $\begin{array}{l}\text { TANS that } \\
\text { responded } \\
\text { only to } \\
\text { light }\end{array}$ & $\begin{array}{l}\text { TANs that } \\
\text { responded } \\
\text { to both }\end{array}$ & Total \\
\hline Putamen & $\begin{array}{l}16 \\
(40.0 \%)\end{array}$ & $\begin{array}{c}8 \\
(20.0 \%)\end{array}$ & $\begin{array}{l}16 \\
(40.0 \%)\end{array}$ & 40 \\
\hline Caudate n. & $\begin{array}{l}10 \\
(17.5 \%)\end{array}$ & $\begin{array}{l}25 \\
(43.9 \%)\end{array}$ & $\begin{array}{l}22 \\
(38.6) \%\end{array}$ & 57 \\
\hline Striatum & $\begin{array}{l}26 \\
(26.8 \%)\end{array}$ & $\begin{array}{l}33 \\
(34.0 \%)\end{array}$ & $\begin{array}{l}38 \\
(39.2 \%)\end{array}$ & 97 \\
\hline
\end{tabular}

The stimuli were presented in alternating recording periods. The data were taken from monkey $R$ after intermission of conditioning and from monkey $D$ during both conditioning periods (see Fig. 1). response to the unrewarded old click. Several days passed before the monkey appeared to realize that no reward came out with the old click and stopped licking in response to it.

Of the 56 TANs recorded, 37 were held long enough during the first set of trials to be tested with both the original and the new clicks (a minimum of $10 \mathrm{~min}$ ). The remaining 19 were tested only with onc stimulus. Seventeen cells (14 of 37 tested with both kinds of stimuli and 3 of 19 tested only with one stimulus) were recorded for more than $20 \mathrm{~min}$, and showed a change in their response patterns to the stimuli. Of all cells tested with both old and new stimuli, seven showed no response and six responded to both kinds of stimuli in the first set of trials. Only four cells responded only to the new stimuli in the first set of trials.

Of the 17 cells for which prolonged recordings were achieved, nine underwent consistent changes in their responsiveness during the extended recordings, with either a gradual acquisition of a response to the new stimulus (six instances) or a weakening of the response to the original stimulus (four instances). We found two instances in which cells changed their response patterns to both stimuli (decrease in response to the old, increase to the new stimulus).

Figure 13 shows the change in activity of one of these TANs, which acquired a conditioned response to the new click sound during the recording sessions on the eighth day of the NEW CONDITIONING task. At the beginning of recording on this day, this neuron showed no response to the new click, although the monkey already had learned that the new click was coupled with reward and licked the spoon efficiently (Fig. 13A, left panel). By contrast, the same neuron responded to the old click, although the monkey had already learned that the old click was uncoupled from reward and showed no licking movements (Fig. $13 B$, left panel). Five minutes after the presentation of the new click (Fig. 13A, middle panel), the neuron showed a slightly suppressed activity in response to the new click, and by $15 \mathrm{~min}$ (Fig. 13A, right panel) the neuron responded with a marked pause followed by a rebound activation. Over the course of the same 10 minute period, the same neuron gradually decreased 
A

before

conditioning

$1-4$ days

5-8 days

9-14 days

15-21 days
PUTAMEN

(80)

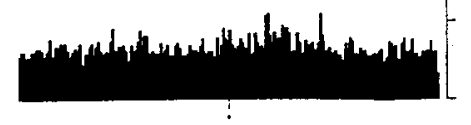

(32)

(29)
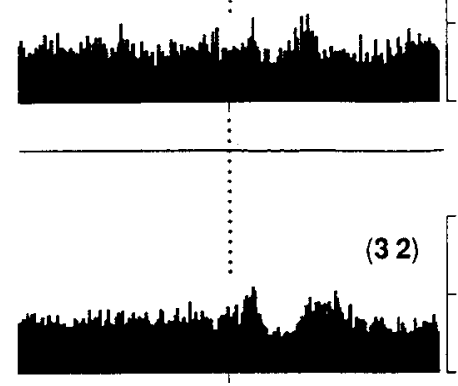

1)
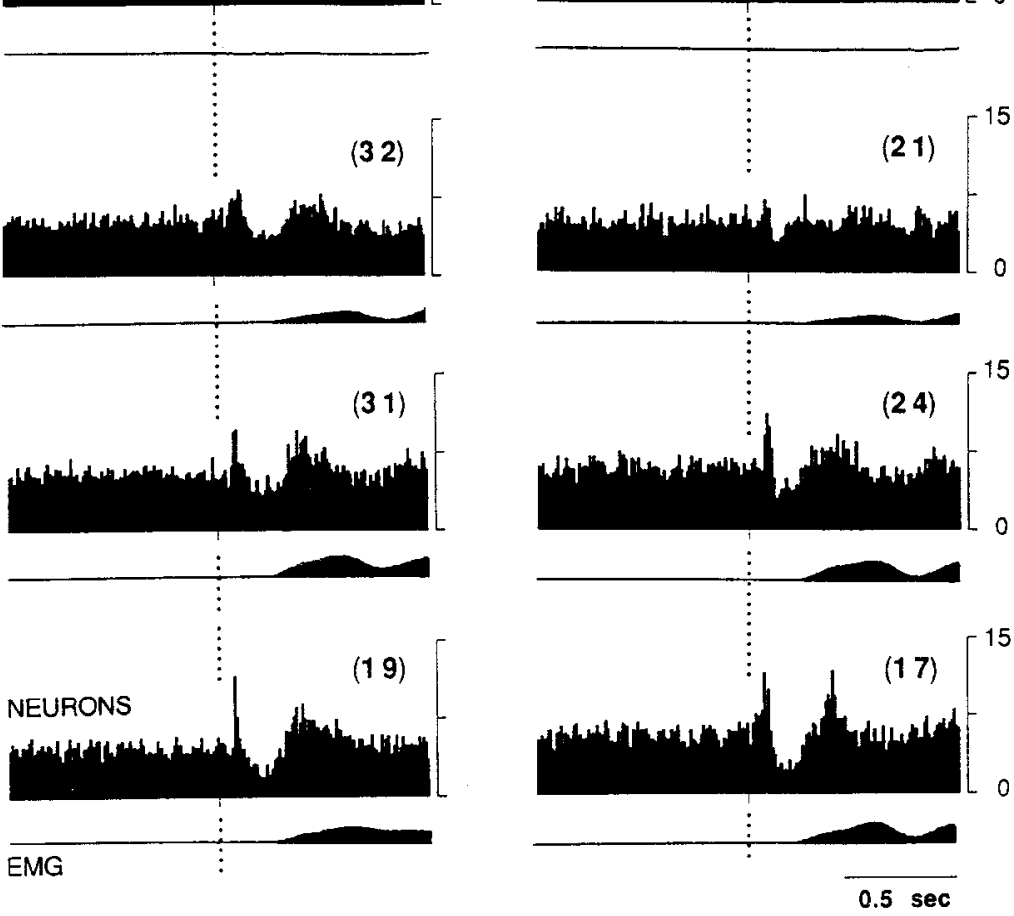

B

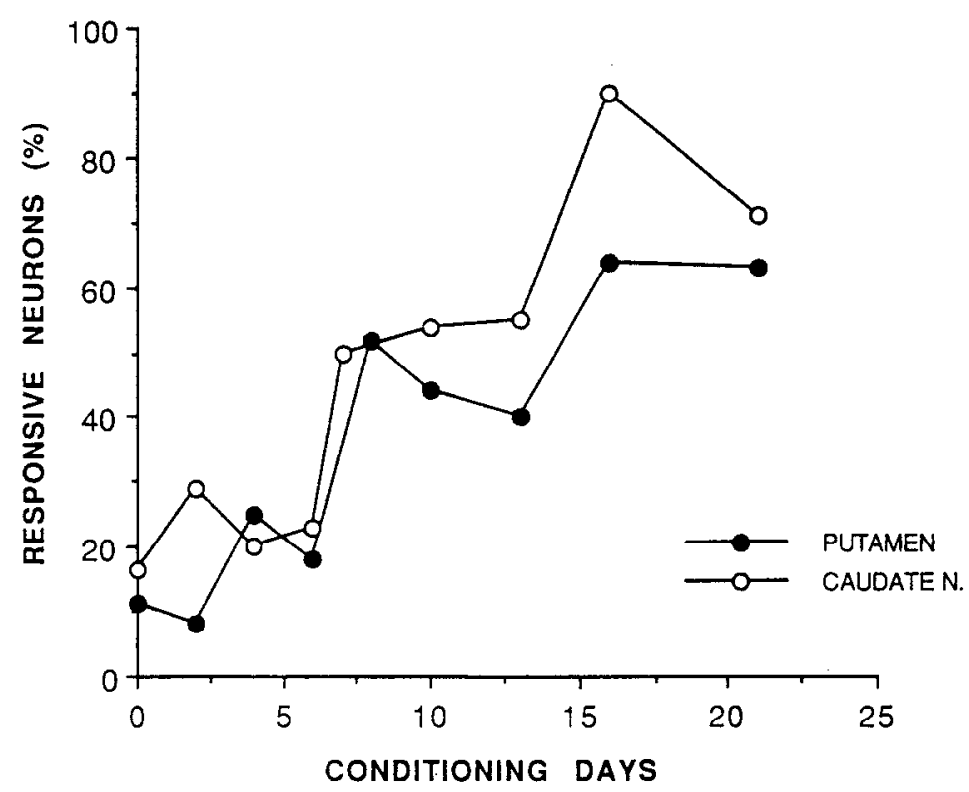

CAUDATE N. imp/sec

(42)
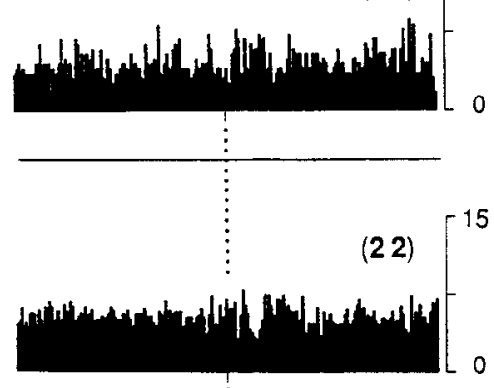

Figure 10. Population responses of TANs to sensory cucs during conditioning in monkey R. $A$, Population histograms constructed by adding histograms from each neuron recorded in the putamen (left) and the caudate nucleus (right) and then dividing the resulting sum by the number of neurons in the samples. Neuronal activity was aligned on the time of click presentation. Recording times before and during conditioning are indicated at the left. Numbers in parentheses indicate the number of TAN records averaged for the population histograms. Averaged EMG records are shown below the histograms. Note that the population response to the conditioning cues gradually became stronger during conditioning. This was true both for the pause in firing and for the initial activation and rebound. $B$, The percentages of TANs responding to the clicks are plotted against time for the caudate nucleus (open circles) and putamen (solid circles). 
Figure 11. Comparison of the percentages of responsive TANs for all TANs recorded from the putamen and the caudate nucleus of the two monkeys before conditioning (open bars), after conditioning (solid bars), and after $\sim 1$ month intermission in training given after overtraining (stippled bars; see Fig. 1). The fractions above each bar show the number of responsive TANs divided by all TANs examined in each experimental condition.

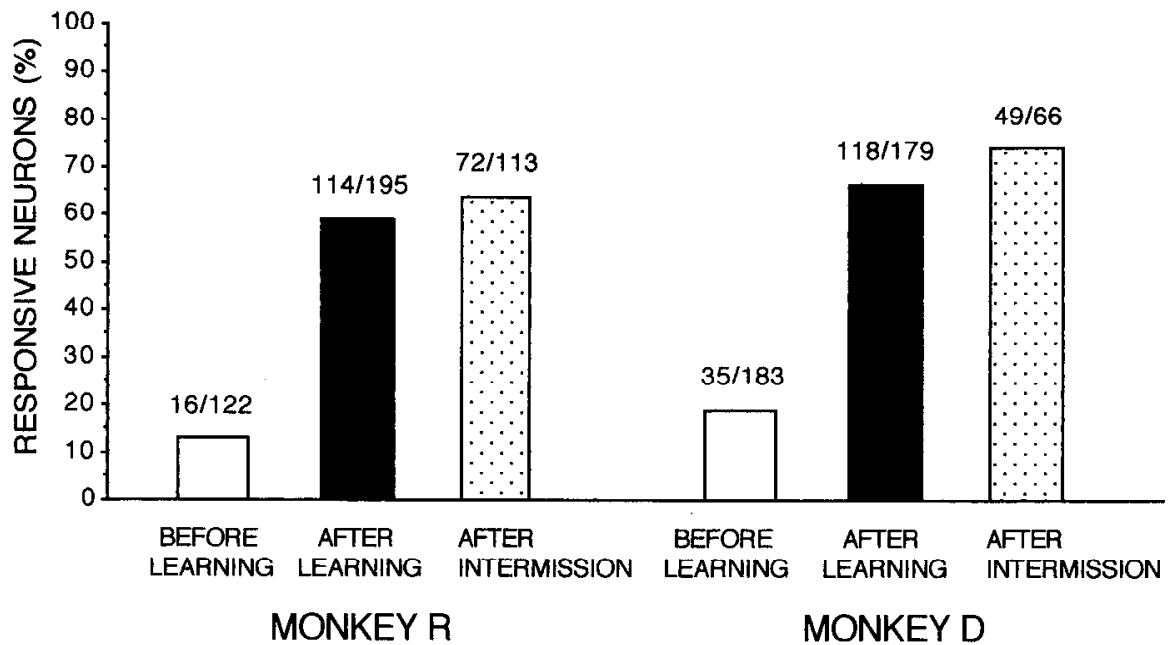

its response to the old click (Fig. $13 B$, middle and right panels). Interestingly, eight out of the 17 cells that could be recorded for an extended period during the NEW CONDITIONING task showed unstable responsiveness. They sometimes responded to the new click, then lost the response in the following trials, and then regained responsivity. It was as though they were in the process of making the kind of switch shown for the neuron illustrated in Figure 13.

\section{Discussion}

In this study we demonstrate that striatal neurons undergo marked changes in responsiveness during the time in which a classical sensorimotor conditioning task is learned by adult macaque monkeys. We tracked the activity of tonically active neurons of the striatum (TANs), which can be identified reliably by their spontaneous tonic activity and which, in monkeys high-

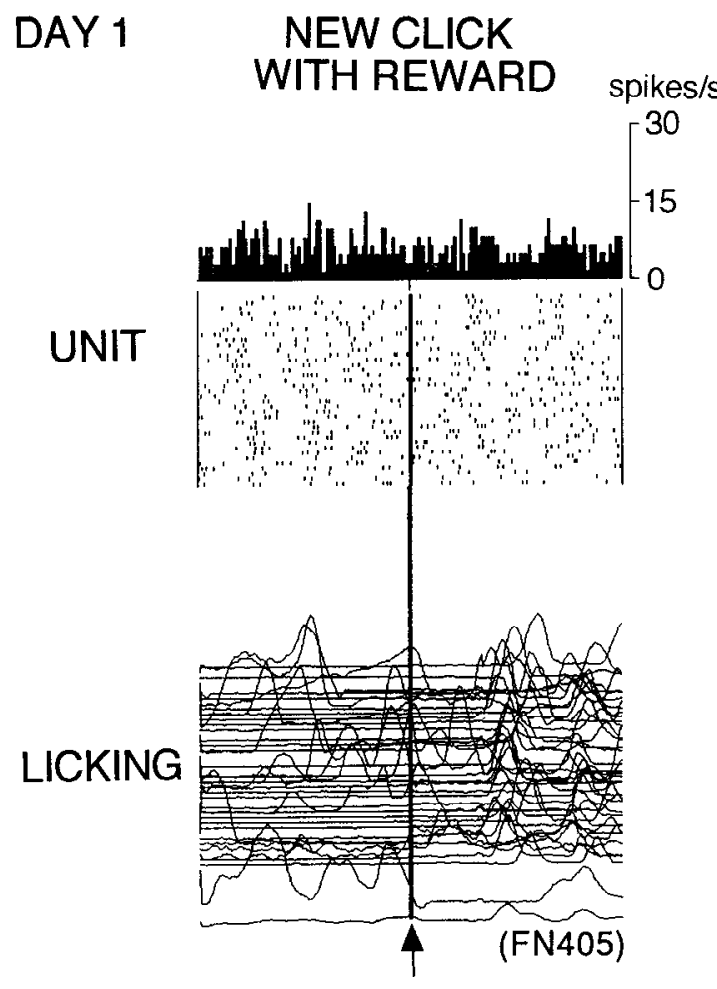

\section{ORIGINAL CLICK WITHOUT REWARD spikes/s}

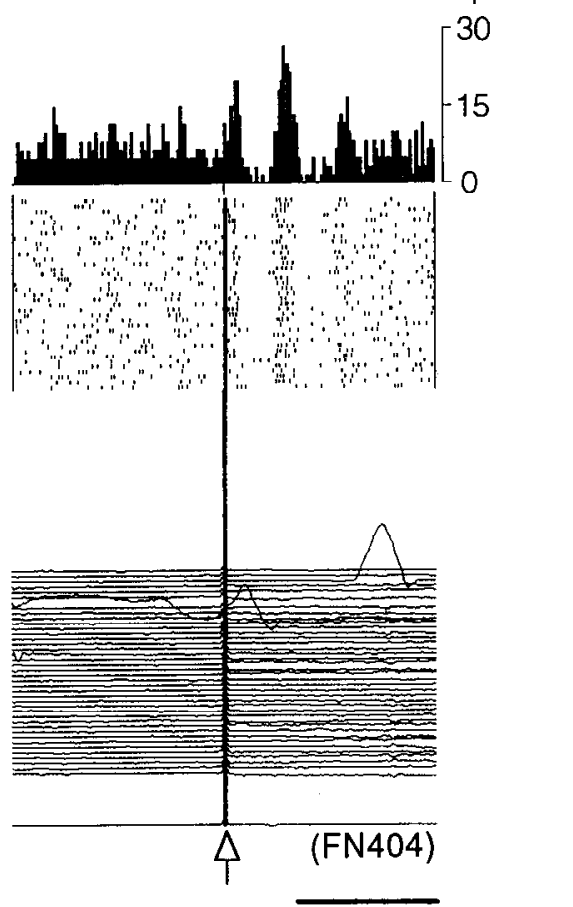

$1 \mathrm{~s}$

Figure 12. Differential response of a TAN to a new click stimulus given with reward and to the original, familiar click stimulus now presented without reward in the NEW CONDITIONING task. The activity was recorded on the first day of the task. Left, The cell emitted no response to the new click, even though the reward was coupled with it. The animal did notice that the reward juice was delivered during this period, and licked the spoon intermittently but ineffectively. Solid arrow indicates the time of the new click and reward presentation. Right, The same TAN showed a strong response to the original click sound, but the monkey made almost no licking movements. The monkey had already noticed by this time during the day's training that no reward came with the original click. Serial recordings of this neuron (not shown) demonstrated that it had an unstable response to the new stimulus about $40 \mathrm{~min}$ later. However, a $5 \mathrm{~min}$ intermission returned the previous nonresponsiveness to the new stimulus. 


\section{A NEW CONDITIONING DAY 8}
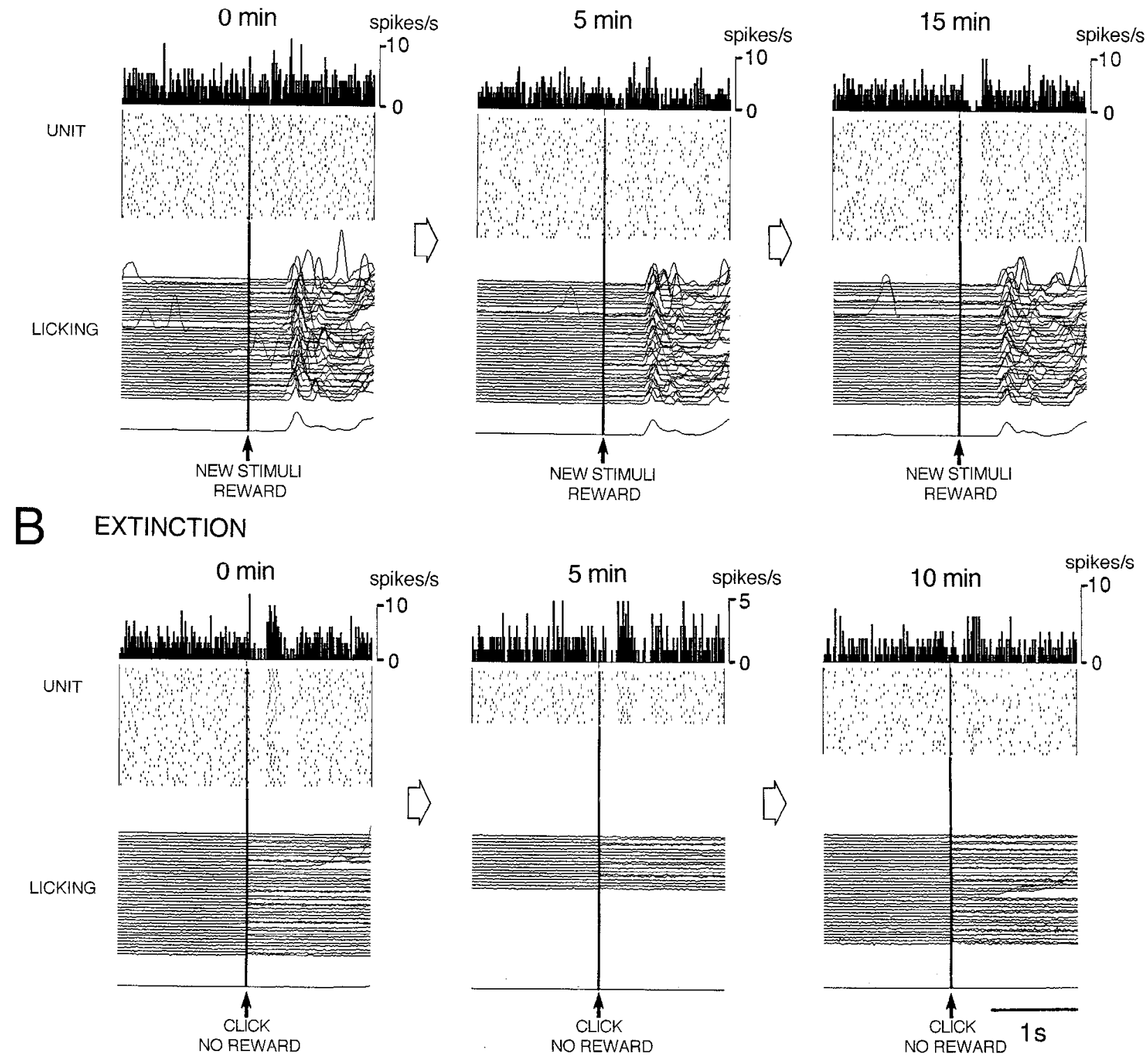

Figure 13. Time course of acquisition and extinction of conditioned response of an individual TAN recorded on the eighth day of the NEW CONDITIONING session. In alternating sets of trials, a novel click was presented with reward delivery $(A)$, and the old click was presented without reward $(B)$. Records were taken over continuous $15 \mathrm{~min}(A)$ and $10 \mathrm{~min}(B)$ periods. Time indicated above each raster display indicates beginning of that set of recordings. $A$, Behaviorally, the monkey (monkey $\mathrm{R}$ ) had learned to lick vigorously to obtain reward juice during the recording periods with the new click. At the start of the recording period (left panel), the TAN recorded showed no response to the new stimulus, but it gradually acquired the response, as shown by records at 5 and at $15 \mathrm{~min}$ (middle and right panels). $B$, By contrast, the same TAN began the recording period showing a response to the old clicks. Behaviorally, the monkey already knew that no reward came with the old clicks, as evidenced by the lack of licking. The records at 5 and 10 min of recording show the gradual loss of the response of the TAN as the extinction trials continued.

ly practiced on such conditioning tasks, respond to the conditioned stimulus in such tasks (Kimura et al., 1984; Kimura, 1986; Apicella et al., 1991). By training monkeys from a completely naive state, we found that TANs responding to the conditioned stimulus before conditioning were relatively rare. The conditioned responsiveness of the TANs as a population was acquired gradually during the course of the behavioral learning, and, once acquired, was maintained for at least a month when training was discontinued and was only gradually extinguished in the absence of reward. Although the population response to the conditioning stimuli took days to emerge, we found that individual TANs could acquire such responses within at least $10 \mathrm{~min}$. This evidence represents the first demonstration of neuronal response plasticity in the primate striatum during behavioral learning.

\section{Relation of the acquired neural responses to reinforcement}

The sensorimotor conditioning task we used was a classical Pavlovian task. Animals were trained to obtain reward juice (unconditioned stimulus) by licking it from a spoon in front of 
their mouths at the time of presentation of a click sound (conditioning stimulus). In the preconditioning period, the reward juice was given separately and independently of reward, so it is natural that the animals evidenced no particular interest in the clicks and did not make licking movements after them. TANs were identified during this period by their characteristic tonic firing pattern $(2-10 \mathrm{~Hz}$ ), wave form (a broad negative spike), and interspike-interval histograms. About 15-20\% responded to the clicks before conditioning. The fact that only a small fraction of the TANs showed responses before the association learning suggests that the response of the TANs to classical conditioning was not a simple sensory response. Without any reinforcement by reward, the external sensory stimulus had the same auditory quality but was without behavioral meaning for the animals. By contrast, when rewards were given simultaneously with the clicks, the animals rapidly came to pay attention to the clicks, and they made more licking movements triggered by the cues. They learned how to obtain the reward efficiently after about a week of training. The numbers of TANs that showed a transient suppression of spontaneous firing in response to the sensory stimuli increased in parallel. The population response of the TANs to the click reached a plateau by 2 weeks both in the putamen and in the caudate nucleus. By this time, more than half of the TANs had become responsive to the conditioned stimulus no matter where in the striatum the neurons were recorded.

The relation of the TAN responses to reinforcement by reward, rather than to the sensory stimulus per se, was supported by the results of extinction trials. In 10 out of 16 cells tested, the neurons lost the response to the clicks when no reward came. In previous cxpcriments, Kimura found that TANs lost their responsiveness instantaneously in NO REWARD sessions (Kimura et al., 1984; Kimura, 1986). By contrast, we observed that the change required many trials in which the clicks were not associated with reward. This difference in findings may be attributable to the different extinction paradigms used in these and in our own experiments. In the previous experiments, REWARD and NO REWARD sessions of 50-60 trials were imposed regularly and alternately for several months. Thus, the monkeys may have learned not to expect the reward in the blocks of NO REWARD trials. In our experiments, by contrast, the rewards and clicks were associated in training carried out for several months, and NO REWARD sessions were only imposed occasionally and at unpredictable times after acquisition of the conditioned behavior. Such a strong influence of expectation on TAN responsivity is in full accord with our findings during the acquisition phase of learning.

It is also clear that the acquired response of the TANs was not related to the licking movements per se. Thus, the TAN responses (1) were not triggered in a time-locked relation to licking, (2) were not somatosensory responses to the tongue's touching the reward juice, and (3) were not responses to the reward itself. Rather, the responses emerged when the external sensory stimulus gained value for the animals as a predictor of reward. Furthermore, as shown in the extinction tests and NEW CONDITIONING task, the responses of the TANs to the clicks declined when the predictive attribute of the clicks no longer obtained. The responses of the TANs appeared to depend critically on the reinforcement association of the sensory stimuli.

Specificity of the acquired sensory responses of TANs

We found a sharp increase in the numbers of TANs responding to the clicks after conditioning. However, not all of the TANs recorded showed the response after learning. What did the other TANs do? We were unable to answer this question, but we did explore the capacity of the TANs to respond to more than conditioning stimulus. We first thoroughly trained the monkeys with clicks as the conditioning stimuli, and then, after acquisition of conditioned behavior, added parallel sessions in which the monkeys were trained to lick in response to LED light stimuli as additional conditioning stimuli. We found that about a quarter of the TANs examined responded only to the clicks, that about a third responded only to the lights, and that the remaining TANs responded to both types of stimulus. The different types were not spatially separated from one another. These results suggest that different sensory inputs can activate different sets of TANs in relation to the learning task, but that different inputs can also converge on populations of TANs. It is possible, then, that individual TANs might have particularized activity profiles in relation to a subset of concurrently presented sensorimotor associations. If so, the population of unresponsive TANs in our sample might have become responsive had we used different cues.

\section{Rapid acquisition of conditioned sensory response by individual TANs during prolonged recordings}

The population response of TANs to the conditioning stimuli appeared gradually during the course of the behavioral conditioning. However, when individual TANs were recorded for extended times during conditioning, we found that a given TAN could acquire the responsiveness in at least as short a time as $10 \mathrm{~min}$. Similarly, we found that individual TANs could lose their responses to the conditioned stimulus after about the same duration of cxtinction training. Remarkably, despitc the fact that the plastic changes could occur over minutes, the acquired response of TANs as a population could be maintained for weeks without training. This suggests that the response plasticity has some of the features associated with long-term memory. TANs are able to register and to store sensory response properties acquired during the course of behavioral learning.

Our experiments do not setlle the question of what the cellular phenotype of the TANs is. TANs are thought to be striatal interneurons, and their physiological characteristics resemble those of the cholinergic neurons of the striatum. In a mapping study of TANs recovered in acute recording experiments (Aosaki et al., 1992, unpublished observations; Kimura et al., 1993), we have found that TANs are preferentially distributed in the striatal matrix, but that a disproportionate number of TANs lie at striosome/matrix borders. This distribution is consistent with the possibility that TANs are, in fact, cholinergic interneurons.

Our experiments also do not settle the question of whether the plasticity of TAN responses reflects processing going on inside the striatum, or is the outcome of modified activity transferred to the striatum from elsewhere, or is representative of both intrinsic and extrinsic modifiability. The striatum receives extensive inputs (Graybiel, 1990; Gerfen, 1992), including projections from regions of the cerebral cortex implicated in attention, movement preparation, and memory (Goldman-Rakic, 1984; Kurata and Wise, 1988a,b; Alexander and Crutcher, 1990a-c; Crutcher and Alexander, 1990; Pardo et al., 1990, 1991; Posner and Petersen, 1990; Mitz et al., 1991; di Pellegrimo and Wise, 1993). The striatum also receives input from the amygdala, which has been implicated in coding the emotional significance of sensory stimuli and in sensory-reward associations (E. A. Gaffan et al., 1988; Cador et al., 1989; Everitt et al., 1989, 1991; D. Gaffan et al., 1989; LeDoux et al., 1990, 
1991; Gaffan and Murray, 1990). Nuclei of the intralaminar thalamus, which themselves receive inputs from reticular activating circuits, also project to the striatum. Any one or more of these sources may contribute to the response plasticity. As discussed below, however, of particular note as a potential source of the documented reward-related activity are the neurons of the substantia nigra pars compacta, which give rise to the dopamine-containing nigrostriatal tract.

\section{Relation between TANs and dopamine-containing neurons of the midbrain}

Schultz and his colleagues have documented a systematic reward-related response plasticity of midbrain dopamine-containing neurons in behaving macaque monkeys (Schultz, 1986. Ljungberg et al., 1992; Schultz et al., 1993). Most of the dopamine-containing neurons fire bursty spike discharges in response to stimuli that attract the attention of the animal. Like TANs, the dopamine-containing neurons do not fire in relation to movements, whether self-initiated or triggered. Stimuli that elicit responses by the dopamine-containing neurons include novel, unexpected stimuli eliciting orienting reactions, and primary reward stimuli such as food or juice. During sensorimotor conditioning, the neurons gradually lose their responsiveness to the primary reward, and they become activated, instead, by the conditioned stimulus (Ljungberg et al., 1992).

At first glance, these responses of substantia nigra pars compacta neurons after learning seem almost mirror images of the TAN responses we report here: both sets of neurons acquire responses to conditioning stimuli, but the responses of the dopamine-containing neurons are transient activations of spike discharge, whereas those of the striatal TANs are transient suppressions. This suggests the interesting possibility that the conditional activity of TANs in the striatum somehow reflects input from the dopamine-containing neurons of the nigral complex.

There are also differences in the response profiles of the striatal and nigral neurons, however. First, the TANs we recorded did not fire to primary reward stimuli, although many were capable of acquiring responses to stimuli predictive of reward. Most TANs also did not appear to respond to novel stimuli. For example, although they did respond more to loud than to soft clicks, they did not respond to a range of other novel sounds. Thus, the activity of the TANs could not simply reflect the activity of the incoming dopamine-containing afferents in all behavioral states, for the great majority of pars compacta neurons do fire to rewarding and novel stimuli. Second, many TANs showed initial activation and rebound activation flanking the suppression of spike discharge. These features (or their inverse) are not regular characteristics of the midbrain neurons' responses to conditioned stimuli as documented by Schultz and coworkers (Ljungberg et al., 1992). Third, the TANs, once having acquired a response to the conditioning stimuli, maintained this response even with prolonged overtraining, when the conditioned behavior became highly automatized. By contrast, Schultz and his colleagues (Ljungberg et al., 1992; Schultz et al., 1993) report that the responses of the dopamine-containing neurons, once acquired, progressively diminish with overtraining. This decreased responsiveness after overtraining was interpreted as parallelling the animals' reduced attention to the conditioned stimulus as the task became automatic and the conditioned stimulus became merely a "temporal reference" for automatic execution of behavior (Ljungberg et al., 1992; Schultz et al., 1993). Habitual occurrence of the conditioned stimuli did not dampen the responsiveness of TANs.
These differences between the conditioned activity of striatal TANs and midbrain dopamine-containing neurons could reflect differences in the behavioral tasks used to test the two populations, but they clearly might, instead, indicate different processing characteristics of nigral and striatal neurons during learning tasks. This latter possibility is quite intriguing. If the dopamine-containing neurons signal attention or vigilance by firing in relation to important sensory stimuli that induce behavioral reactions and sensorimotor learning, but then lose this responsiveness, this information must not only be transferred during the learning process to motor control pathways, but also must then be stored so that the conditioned behavior can actually be executed. Our results suggest that, in the striatum, TANs can acquire and maintain a record of the conditioned sensory responsiveness for prolonged periods of time. The TANs of the striatum may thus be part of a mechanism active in the acquisition and retention of sensorimotor associations.

\section{References}

Alexander GE, Crutcher MD (1990a) Functional architecture of basal ganglia circuits: neural substrates of parallel processing. Trends Neurosci 13:266-272.

Alexander GE, Crutcher MD (1990b) Preparation for movement: neural representations of intended direction in three motor areas of the monkey. J Neurophysiol 64:133-150.

Alexander GE, Crutcher MD (1990c) Neural representations of the target (goal) of visually guided arm movements in three motor areas of the monkey. J Neurophysiol 64:164-178.

Aosaki T, Kimura M, Graybiel AM (1992) Physiologically identified tonically active neurons of primate striatum lie in the matrix compartment. IBAGS 4th Congr Abstr 3.

Aosaki T, Tsubokawa H, Watanabe K, Graybiel AM, Kimura M (1993) Tonically active neurons in the primate striatum acquire responses to sensory stimuli during behavioral conditioning. Soc Neurosci Abstr 19:1585.

Apicella P, Scarnati E, Schultz W (1991) Tonically discharging neurons of monkey striatum respond to preparatory and rewarding stimuli. Exp Brain Res 84:672-675.

Cador M, Robbins TW, Everitt BJ (1989) Involvement of the amygdala in stimulus-reward associations: interaction with the ventral striatum. Neuroscience 30:77-86.

Carli M, Evenden JL, Robbins TW (1985) Depletion of unilateral striatal dopamine impairs initiation of contralateral actions and not sensory attention. Nature 313:679-682.

Cools AR (1980) Role of the neostriatal dopaminergic activity in sequencing and selecting behavioural strategies: facilitation of processes involved in selecting the best strategy in a stressful situation. Behav Brain Res 1:361-378.

Crutcher MD, Alexander GE (1990) Movement-related neuronal activity selectively coding either direction or muscle pattern in three motor areas of the monkey. J Neurophysiol 64:151-163.

DeLong MR, Georgopoulos AP (1981) Motor functions of the basal ganglia. In: Handbook of physiology (Brooks VB, ed), pp 1017-1061. Bethesda, MD: American Physiological Society.

di Pellegrimo G, Wise SP (1993) Visuospatial versus visuomotor activity in the premotor and prefrontal cortex of a primate. $J$ Neurosci 13:1227-1243.

Evarts EV, Kimura M, Wurtz RH, Hikosaka O (1984) Behavioral correlates of activity in basal ganglia neurons. Trends Neurosci 7:447453.

Everitt BJ, Cador M, Robbins TW (1989) Interactions between the amygdala and ventral striatum in stimulus-reward associations: studies using a second-order schedule of sexual reinforcement. Neuroscience 30:63-75.

Everitt BJ, Morris KA, O'Brien A, Robbins TW (1991) The basolateral amygdala-ventral striatal system and conditioned place preference: further evidence of limbic-striatal interactions underlying rewardrelated processes. Neuroscience 42:1-18.

Gaffan D, Murray EA (1990) Amygdalar interaction with the mediodorsal nucleus of the thalamus and the ventromedial prefrontal cortex in stimulus-reward associative learning in the monkey. $J$ Neurosci $10: 3479-3493$ 
Gaffan D, Gaffan EA, Harrison S (1989) Visual-visual associative learning and reward-association learning in monkeys: the role of the amygdala. J Neurosci 9:558-564.

Gaffan EA, Gaffan D, Harrison S (1988) Disconnection of the amygdala from visual association cortex impairs visual reward-association learning in monkeys. J Neurosci 8:3144-3150.

Gerfen CK (1992) The neostriatal mosaic: multiple levels of compartmental organization. Trends Neurosci 15:133-139.

Goldman-Rakic PS (1984) Modular organization of prefrontal cortex. Trends Neurosci 7:417-422.

Graybiel AM (1990) Neurotransmitters and neuromodulators in the basal ganglia. Trends Neurosci 13:244-254.

Kimura M (1986) The role of primate putamen neurons in the association of sensory stimuli with movement. Neurosci Res 3:436443.

Kimura M (1992) Behavioral modulation of sensory responses of primate putamen neurons. Brain Res 578:204-214.

Kimura M, Rajkowski J, Evarts E (1984) Tonically discharging putamen neurons exhibit set-dependent responses. Proc Natl Acad Sci USA 81:4998-5001.

Kimura M, Kato M, Shimazaki H (1990) Physiological properties of projection neurons in the monkey striatum to the globus pallidus. Exp Brain Res 82:672-676.

Kimura M, Aosaki T, Graybiel AM (1993) Differential localization of tonically active neurons of primate striatum in the matrix and at striosome/matrix boundaries. Soc Neurosci Abstr 19:1585.

Kurata K, Wise SP (1988a) Premotor cortex of rhesus monkeys: setrelated activity during two conditional motor tasks. Exp Brain Res 69:327-343.

Kurata K, Wise SP (1988b) Premotor and supplementary motor cortex in rhesus monkeys: neuronal activity during externally- and internally-instructed motor lasks. Exp Brain Res 72:237-248.

Kusama T, Mabuchi M (1970) Stereotaxic atlas of the brain of $\mathrm{Macaca}$ fuscata. Tokyo: University of Tokyo; Baltimore, MD: University Park.
LcDoux JE, Cicchetti P, Xagoraris A, Romanski LM (1990) The lateral amygdaloid nucleus: sensory interface of the amygdala in fear conditioning. J Neurosci 10:1062-1069.

LeDoux JE, Farb CR, Romanski LM (1991) Overlapping projections to the amygdala and striatum from auditory processing areas of the thalamus and cortex. Neurosci Lett 134:139-144.

Ljungberg T, Apicella P, Schultz W (1992) Responses of monkey dopamine neurons during learning of behavioral reactions. J Neurophysiol $67: 145-163$.

Mitz AR, Godschalk M, Wise SP (1991) Learning-dependent neuronal activity in the premotor cortex: activity during the acquisition of conditional motor associations. J Neurosci 11:1855-1872.

Pardo JV, Pardo PJ, Janer KW, Raichle ME (1990) The anterior cingulate cortex mediates processing selection in the Stroop attentional conflict paradigm. Proc Natl Acad Sci USA 87:256-259.

Pardo JV, Fox PT, Raichle ME (1991) Localization of a human system for sustained attention by positron emission tomography. Nature 349: 61-64.

Posner MI, Petersen SE (1990) The attention system of the human brain. Annu Rev Neurosci 13:25-42.

Schultz W (1986) Responses of midbrain dopamine neurons to behavioral trigger stimuli in the monkey. J Neurophysiol 56:1439-1461.

Schultz W, Apicella P, Ljungberg T (1993) Responses of monkey dopamine neurons to reward and conditioned stimuli during successive steps of learning a delayed response task. J Neurosci 13:900913.

Seitz RJ, Roland E, Bohm C, Greitz T, Stone ES (1990) Motor learning in man: a positron emission tomographic study. Neuroreport 1:5760.

Suzuki H, Azuma M (1976) A glass-insulated "elgiloy" microelectrode for recording unit activity in chronic monkey experiments. Electroencephalogr Clin Neurophysiol 41:93-95. 